\title{
Nitric oxide donor sodium nitroprusside-induced transcriptional changes and hypocrellin biosynthesis of Shiraia sp. S9
}

\author{
Yan Jun Ma ${ }^{1,2}$, Xin Ping Li ${ }^{1}$, Yue Wang ${ }^{1}$ and Jian Wen Wang ${ }^{1 *}$ (D)
}

\begin{abstract}
Background: Nitric oxide (NO) is a ubiquitous signaling mediator in various physiological processes. However, there are less reports concerning the effects of $\mathrm{NO}$ on fungal secondary metabolites. Hypocrellins are effective anticancer photodynamic therapy (PDT) agents from fungal perylenequinone pigments of Shiraia. NO donor sodium nitroprusside (SNP) was used as a chemical elicitor to promote hypocrellin biosynthesis in Shiraia mycelium cultures.

Results: SNP application at 0.01-0.20 mM was found to stimulate significantly fungal production of perylenequinones including hypocrellin A (HA) and elsinochrome A (EA). SNP application could not only enhance HA content by $178.96 \%$ in mycelia, but also stimulate its efflux to the medium. After 4 days of SNP application at $0.02 \mathrm{mM}$, the highest total production (110.34 mg/L) of HA was achieved without any growth suppression. SNP released NO in mycelia and acted as a pro-oxidant, thereby up-regulating the gene expression and activity of reactive oxygen species (ROS) generating NADPH oxidase (NOX) and antioxidant enzymes, leading to the increased levels of superoxide anion $\left(\mathrm{O}_{2}{ }^{-}\right)$ and hydrogen peroxide $\left(\mathrm{H}_{2} \mathrm{O}_{2}\right)$. Gene ontology $(\mathrm{GO})$ analysis revealed that SNP treatment could up-regulate biosynthetic genes for hypocrellins and activate the transporter protein major facilitator superfamily (MFS) for the exudation. Moreover, SNP treatment increased the proportion of total unsaturated fatty acids in the hypha membranes and enhanced membrane permeability. Our results indicated both cellular biosynthesis of HA and its secretion could contribute to HA production induced by SNP.
\end{abstract}

Conclusions: The results of this study provide a valuable strategy for large-scale hypocrellin production and can facilitate further understanding and exploration of $\mathrm{NO}$ signaling in the biosynthesis of the important fungal metabolites.

Keywords: Shiraia, Sodium nitroprusside, Nitric oxide, Hypocrellin, Oxidative stress

\section{Background}

Shiraia bambusicola P. Hennings is a bambusicolous fungus parasitized on bamboo tender twigs and its fruiting bodies have been used in traditional Chinese medicine to stimulate blood circulation, relieve expectoration, cure rheumarthritis and relax muscle rigidity $[1,2]$. Hypocrellins, main perylenequinone pigments

\footnotetext{
*Correspondence: jwwang@suda.edu.cn; bcjwwang@gmail.com

${ }^{1}$ College of Pharmaceutical Sciences, Soochow University, Suzhou 215123, China

Full list of author information is available at the end of the article
}

isolated from the hypha and fruit-bodies of Shiraia fungi, have being developed as new non-porphyrin and reactive oxygen species (ROS)-generating photosensitizers in photodynamic therapy (PDT) for clinical application of anti-microbes, -cancers and -viruses $[1,3,4]$. Due to the difficulty of chemical synthesis of hypocrellins and the scarcity of wild fruiting bodies [5], Shiraia mycelium culture has become a promising production process of this new PDT agent [6]. More process strategies were applied to enhance hypocrellin production in the culture, including the condition optimization for the cultures [7], and application of

(C) The Author(s) 2021. This article is licensed under a Creative Commons Attribution 4.0 International License, which permits use, sharing, adaptation, distribution and reproduction in any medium or format, as long as you give appropriate credit to the original author(s) and the source, provide a link to the Creative Commons licence, and indicate if changes were made. The images or other third party material in this article are included in the article's Creative Commons licence, unless indicated otherwise in a credit line to the material. If material is not included in the article's Creative Commons licence and your intended use is not permitted by statutory regulation or exceeds the permitted use, you will need to obtain permission directly from the copyright holder. To view a copy of this licence, visit http://creativeco mmons.org/licenses/by/4.0/. The Creative Commons Public Domain Dedication waiver (http://creativecommons.org/publicdomain/ zero/1.0/) applies to the data made available in this article, unless otherwise stated in a credit line to the data. 
biotic $[8,9]$ or abiotic elicitors [10, 11]. Nitric oxide (NO), a small molecular signal, was found to be involved in the induced hypocrellin production by a fungal elicitor from Aspergillus niger at $50 \mu \mathrm{g} / \mathrm{mL}$ [12] and a fungal elicitor PB90 at $5 \mathrm{nM}$ [8]. The endogenous NO in S. bambusicola was also observed in extractive culture of Shiraia by Triton X-100 [13].

$\mathrm{NO}$ is an important regulatory molecule in mammals [14]. Recently, NO has been proved to be an essential signal in the elicitation of plant defense and secondary metabolite biosynthesis [15]. NO released by a NO donor sodium nitroprusside (SNP) could elicit plant secondary metabolites such as marjoram essential oil [16], total phenols and flavonoids in Echinacea purpurea roots [17], artemisinin in Artemisia annua hairy roots [18], and taxol in Taxus yunnanensis cells [19]. However, there are few reports on the effects of NO on fungal growth and secondary metabolism. NO may mediate L-arginine-induced conidiation of Coniothyrium minitans [20]. Wang and Higgins (2005) reported that the germination and development of Colletotrichum coccodes conidia were significantly inhibited by SNP at $100 \mu \mathrm{M}$ [21]. A NO-releasing compound diethylenetriamine-NoNoate at $1.5 \mathrm{mM}$ increased drastically the formation of cleistothecia in A. nidulans, suggesting a positive regulator of $\mathrm{NO}$ on fungal sexual development [22]. Zhao et al. demonstrated that NO was involved in the co-culture of Inonotus obliquus with Phellinus morii and triggered the biosynthetical pathway of phenylpropanoids, leading to an increased production of styrylpyrone derivatives [23]. SNP at $5 \mathrm{mM}$ increased yield of ganoderic triterpenoid by 40.94\% in submerged fermenting Ganoderma lucidum [24]. Although the endogenous NO generation was observed in S. bambusicola treated by fungal elicitors $[8,12]$ and Triton $\mathrm{X}-100[13]$, the regulation roles of NO on fungal hypocrellin biosynthesis are still undetermined. Therefore, as a follow-up to our efforts on enhancing hypocrellin production $[9,25]$ and elucidating the role of $\mathrm{NO}$ on secondary metabolite biosynthesis [13, 26], we examined the effects of SNP on Shiraia hypocrellin biosynthesis. We also investigated the relationship between $\mathrm{NO}$ and elicitation responses including ROS generation, the activation of antioxidant defenses and hypocrellin production in Shiraia mycelium cultures. In this study, de novo transcriptome sequencing for Shiraia sp. S9 was performed for better understanding the NO regulation on hypocrellin biosynthesis. This study presented a novel elicitor of hypocrellin production and shed light on the relational roles of $\mathrm{NO}$ on the biosynthesis of secondary metabolites.

\section{Results}

SNP application released NO and enhanced hypocrellin production

After the application of SNP at $0.10 \mathrm{mM}$ to 3-day-old cultures, we observed a rise of green fluorescence of $\mathrm{NO}$ indicator 4, 5-diaminofluorescein diacetate (DAF2-DA) in Shiraia mycelia, whereas the relative fluorescent ration was markedly decreased by $\mathrm{NO}$ scavenger 2-(4-carboxyphenyl)-4, 4, 5, 5-tetramethylimidazoline1-oxyl-3-oxide (cPTIO), indicating the release of $\mathrm{NO}$ in hyphae (Fig. 1a, b). On the solid medium, the concentration of fungal conidia was decreased from $17.08 \times 10^{6}$ to $2.43 \times 10^{6}$ spores $/ \mathrm{mL}$ by SNP treatment (Fig. $2 \mathrm{a}$ ). The pycnidium formation was suppressed by SNP (Fig. 2b). Although SNP at higher concentration $(1.00 \mathrm{mM})$ inhibited the fungal growth, the diameter of fungal colony was increased on day 6 by SNP at 0.01 or $0.02 \mathrm{mM}$ (Fig. 2c, d). In the liquid culture, there were not any notable changes in fungal biomass (Additional file 1: Figure S1A), $\mathrm{pH}$ value and sugar consumption (Additional file 1: Figure S2) in medium after SNP application at $0.10 \mathrm{mM}$. However, SNP decreased fungal pellet diameter by 16.09 25.73\% (Additional file 1: Figure S1B) and the pellets were colored with dark red (Fig. 2e). The influence of SNP on hypocrellins including hypocrellin A (HA) and $\mathrm{HC}$, and elsinochrome (A, B and $\mathrm{C}$ ) in the culture was detected by HPLC (Additional file 1: Figure S3). Both the intracellular HA (1.22-fold of control) and EA (5.88-fold of control) were enhanced by SNP at $0.10 \mathrm{mM}$ (Table 1). The extracellular HA was increased by $87.56 \%$, while HC, EB and $\mathrm{EC}$ were not detected in cultural broth with or without SNP treatment.

As HA is a major bioactive hypocrellin constituent in Shiraia [9], we optimized the conditions (concentration and addition time) of SNP application to obtain higher HA production. SNP at lower concentrations (0.01-0.20 mM) had no obvious impacts on fungal biomass (Additional file 1: Figure S4A), but increased HA contents in mycelium and in cultural broth. The higher HA in mycelium $(9.72 \mathrm{mg} / \mathrm{g}$ DW) and in cultural broth $(4.64 \mathrm{mg} / \mathrm{L})$ were obtained at $0.02 \mathrm{mM}$ and $0.20 \mathrm{mM}$, respectively (Additional file 1: Figure S4B, C). When SNP at $0.02 \mathrm{mM}$ was applied on different days, the higher contents of intracellular HA $(9.97 \mathrm{mg} / \mathrm{g}$ DW $)$ and the released HA in cultural broth $(4.42 \mathrm{mg} / \mathrm{L})$ were achieved on day 3 (Additional file 1: Figure S5). Hence, SNP application at $0.02 \mathrm{mM}$ on day 3 of the culture was then used for subsequent culture. The time course of the effect of SNP on HA production in 9-day-old cultures was shown in Fig. 3. Under this optimal condition, SNP application did not suppress mycelial growth (Fig. 3a), but promoted the intracellular HA contents by $73.31-178.96 \%$ (Fig. 3b) and extracellular HA accumulation by $26.09-119.26 \%$ 
a

Control
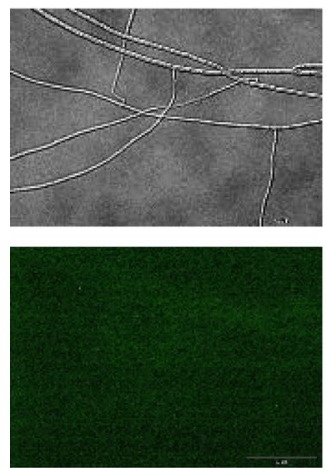

b
$\mathrm{SNP}+\mathrm{cPTIO}$
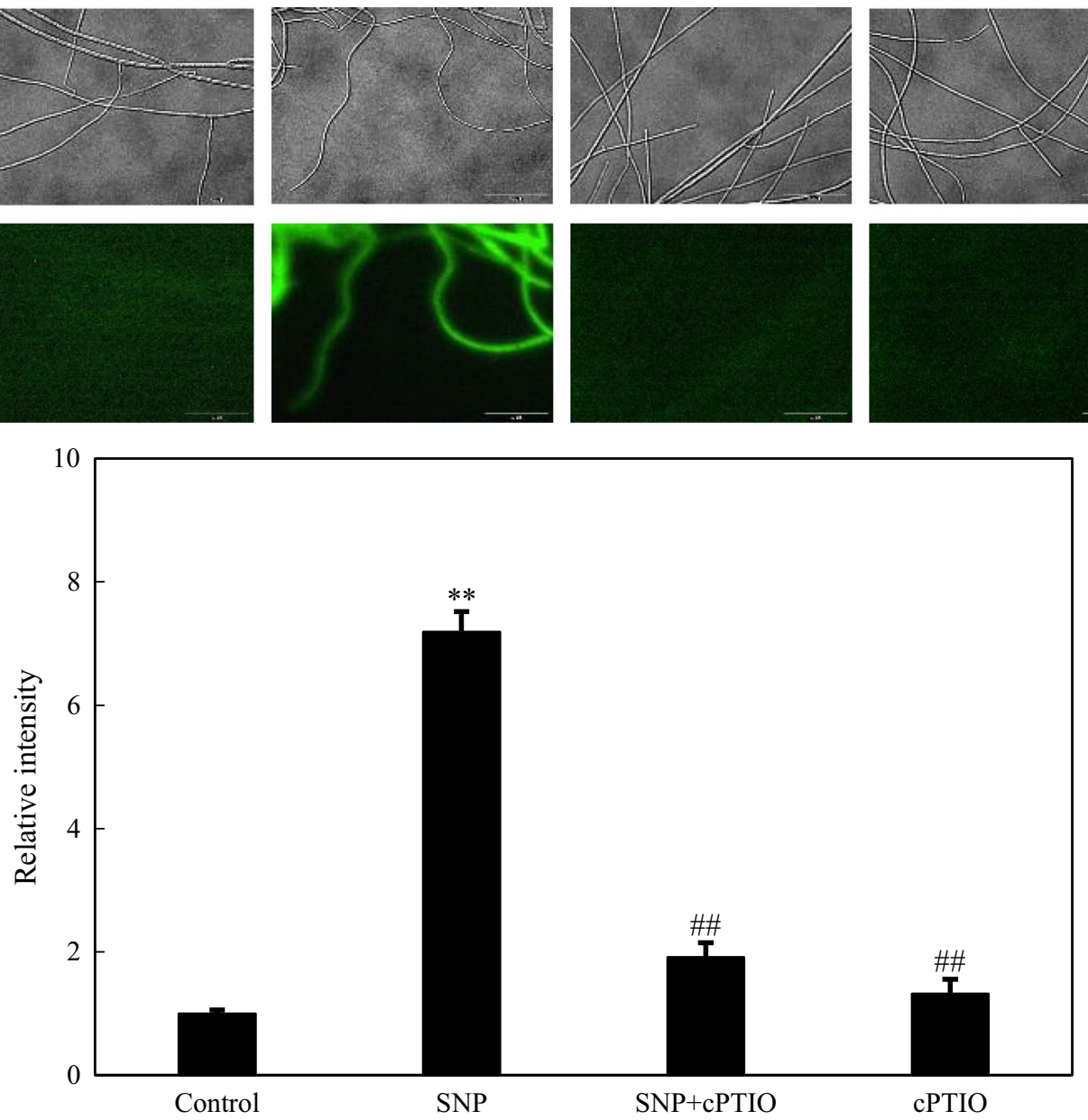

Fig. 1 NO generation in SNP-treated mycelia of Shiraia sp. S9. a Bright-field image (above) and fluorescence microscopy of DAF-2-DA-stained mycelium (below) in cultures. SNP was added at $0.10 \mathrm{mM}$ on day 3 of the culture. CPTIO $(0.10 \mathrm{mM})$ was added 30 min prior to SNP treatment. The photos were taken after $2 \mathrm{~h}$ of SNP treatment. $\mathbf{b} \mathrm{NO}$ accumulation (relative intensity of fluorescent ratio) in mycelium after SNP treatment. Values are mean \pm SD from three independent experiments $\left({ }^{* *} p<0.01\right.$ vs. control, ${ }^{\#} p<0.01$ vs. SNP group)

(Fig. 3c). The total HA production was enhanced to $110.34 \mathrm{mg} / \mathrm{L}$ on day 7 , a 2.65 -fold increase over the control without SNP addition (Fig. 3d).

\section{SNP-induced transcriptional changes of genes for hypocrellin biosynthesis}

To examine the transcriptional changes of Shiraia sp. S9 after SNP treatment, RNA-Seq experiment was subsequently performed. There were altogether 84,275 unigenes assembled with an average length of 1,011.81 bp (base pairs) and an N50 of 5,399 bp (Additional file 1: Table S1, Figure S6). 98.62\%, 54.40\%, 37.37\%, 30.86\% and $90.40 \%$ of the total unigenes were resemble to known genes reported in the current databases in Additional file 1: Table S2. A total of 571 differentially expressed genes (DEGs) were identified (Additional file 2: Table S3), including up-regulated unigenes $(355,62.17 \%)$ and down-regulated unigenes (216, 37.83\%) under SNP treatment (Additional file 1: Figure S7). These DEGs were categorized into the independent classification group "biological process (BP)", "molecular function (MF)" and "cellular component (CC)" (Additional file 2: Table S4). Compared with the annotated unigenes of BP (270 DEGs) and CC (169 DEGs) (Additional file 1: Figure S8A, B), more than 322 DEGs were categorized into MF group (Additional file 1: Figure S8C). Within the MF category, DEGs (129 unigenes) assigned to 'catalytic activity' (GO:0003824) were of the highest proportion and other DEGs were mainly involved in 'oxidoreductase activity' (GO:0016491), 'transporter activity' (GO:0005215) 


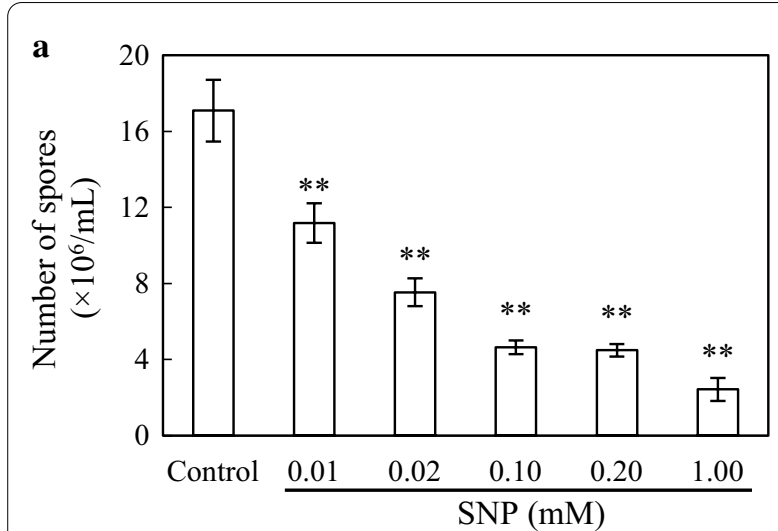

c

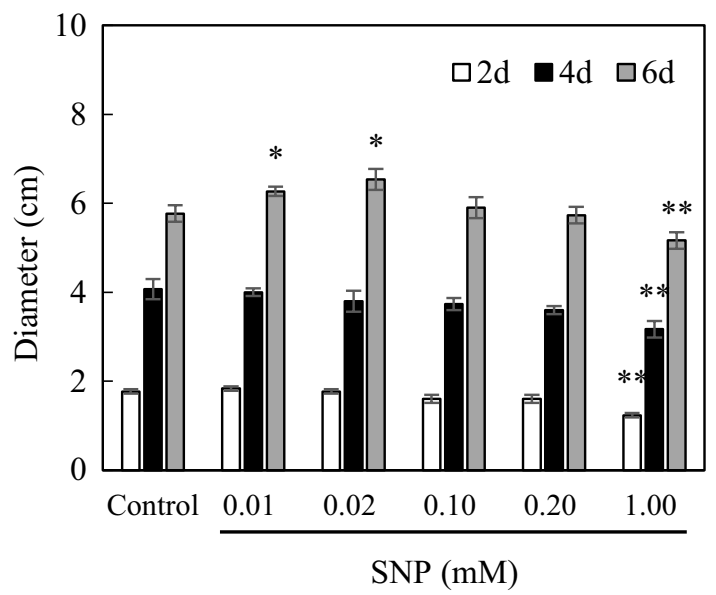

b

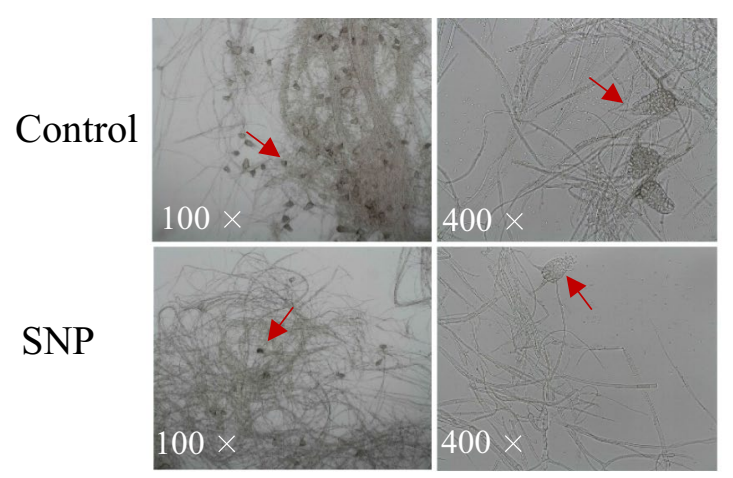

d

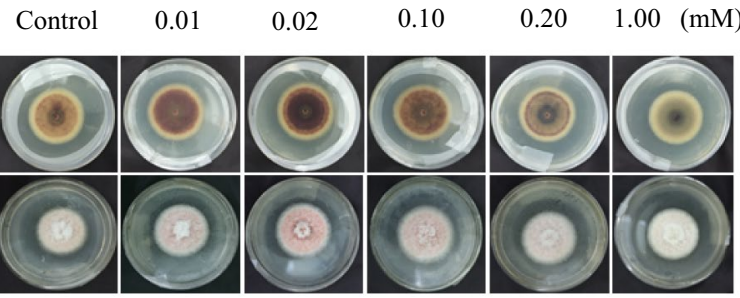

e

Control

SNP

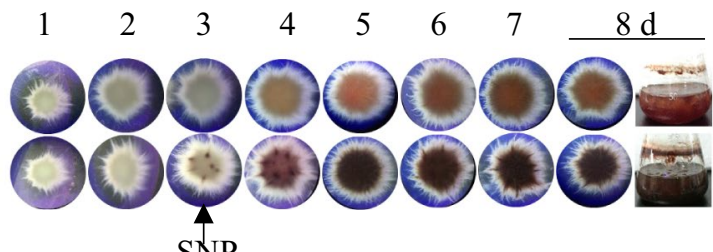

Fig. 2 Effects of SNP on the growth and development of Shiraia sp. S9. a Effect of SNP (0.01-1.00 mM) on the generation of conidia of S9 strain on day 8 in solid medium culture. $\mathbf{b}$ The morphologic characteristics of S9 strain was kept on PDA with or without SNP (0.10 mM) treatment for 8 days. c The diameter of S9 colony treated by SNP at 0.01-1.00 mM for 2- 6 days. $\mathbf{d}$ The colony morphology of S9 strain with SNP (0.01-1.00 mM) treatment for 6 days. e Pellet morphology $(15 \times)$ in submerged culture of 59 strain under SNP treatment at $0.10 \mathrm{mM}$ on day 3. The culture was maintained in $150-\mathrm{mL}$ flask containing $50 \mathrm{~mL}$ of the liquid medium at $150 \mathrm{rpm}$ and $28^{\circ} \mathrm{C}$. The red arrow indicates pycnidium. The black arrow indicates addition time. Values are mean \pm SD from three independent experiments $\left({ }^{*} p<0.05\right.$ and ${ }^{* *} p<0.01$ vs. control)

Table 1 Effects of SNP on the individual hypocrellin production in submerged cultures of Shiraia sp. S9

\begin{tabular}{|c|c|c|c|c|c|}
\hline $\mathrm{PQ}$ production $(\mathrm{mg} / \mathrm{L})$ & EC & EB & $\mathrm{HC}$ & EA & HA \\
\hline \multicolumn{6}{|c|}{ Intracellular PQs in mycelia } \\
\hline Control & $0.54 \pm 0.05$ & $1.03 \pm 0.13$ & $5.68 \pm 1.90$ & $1.93 \pm 0.04$ & $47.05 \pm 3.30$ \\
\hline SNP & $0.50 \pm 0.03$ & $1.29 \pm 0.23$ & $7.38 \pm 0.29$ & $11.34 \pm 0.78^{* *}$ & $57.54 \pm 3.50^{*}$ \\
\hline \multicolumn{6}{|c|}{ Extracellular PQs in cultural broth } \\
\hline Control & ND & ND & ND & $0.13 \pm 0.04$ & $2.25 \pm 0.20$ \\
\hline SNP & ND & ND & ND & $0.14 \pm 0.02$ & $4.22 \pm 0.40^{* *}$ \\
\hline
\end{tabular}

The SNP at $0.10 \mathrm{mM}$ was added into the mycelium cultures on day 3 and the culture was maintained in $150-\mathrm{mL}$ flask containing $50 \mathrm{~mL}$ of the liquid medium at $150 \mathrm{rpm}$ and $28^{\circ} \mathrm{C}$ for 8 days. Values are mean \pm SD from three independent experiments $\left({ }^{*} p<0.05\right.$ and ${ }^{* *} p<0.01$ vs. control). ND indicates no detection

and 'transmembrane transporter activity' (GO:0022857) items.

Based on the reported gene clusters for hypocrellin biosynthesis [27-29], we explored the expression changes of DEGs associated with hypocrellin biosynthesis under SNP treatment. De novo sequencing and comparative analysis revealed 113 putative DEGs were enriched in 10 items related to fungal hypocrellin production (Additional file 2: Table S5 and S6), including 'polyketide synthase', 'hydroxylase', 

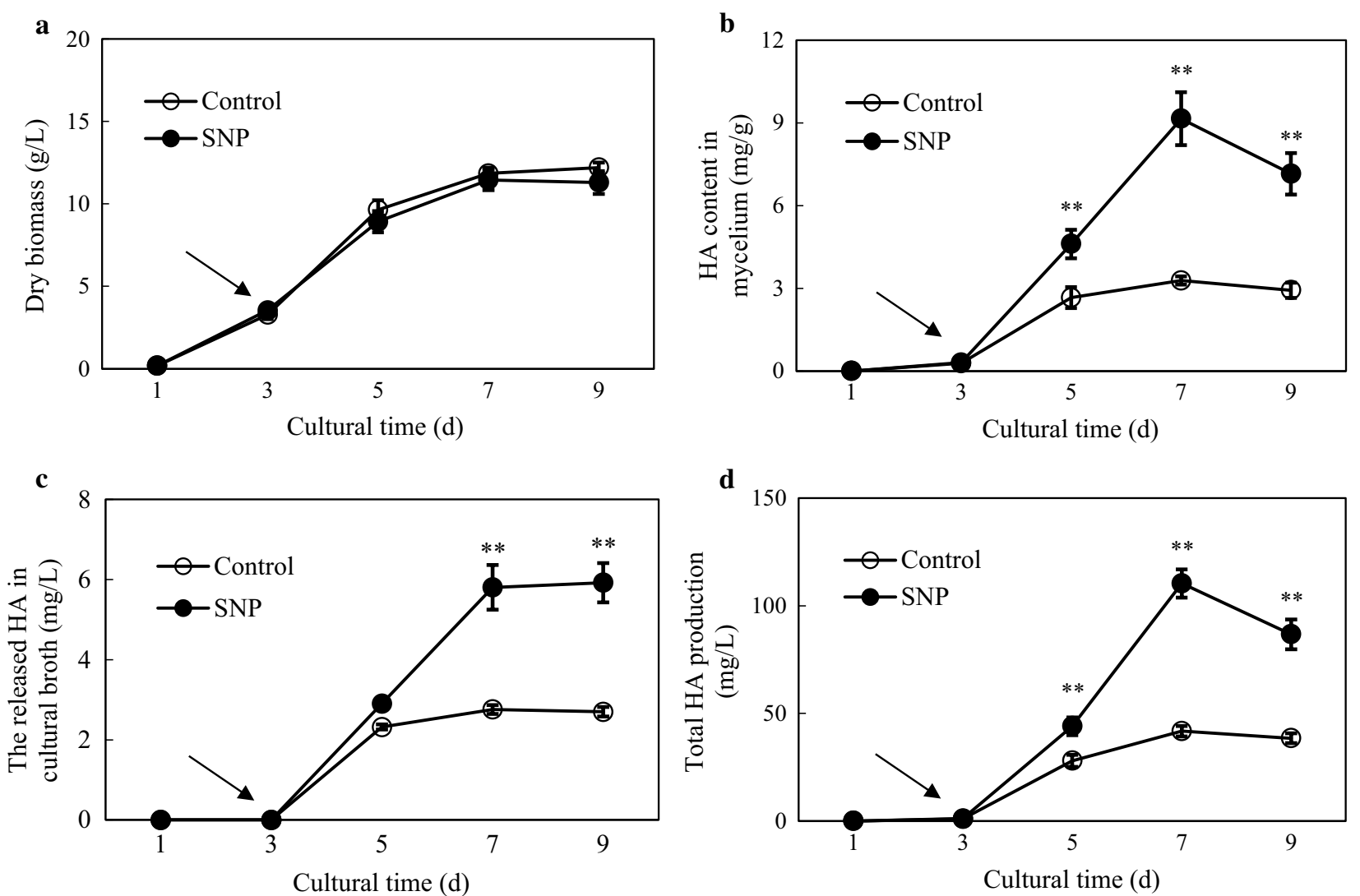

Fig. 3 Time profiles of fungal biomass $\mathbf{a}$, HA content in mycelia $\mathbf{b}$, the released HA in cultural broth $\mathbf{c}$ and total HA production $\mathbf{d}$ in submerged culture of Shiraia sp. S9 under $0.02 \mathrm{mM}$ SNP treatment on day 3. The culture was maintained in 150-mL flask containing $50 \mathrm{~mL}$ of the liquid medium at $150 \mathrm{rpm}$ and $28^{\circ} \mathrm{C}$. The arrow represents the time of SNP addition. Values are mean \pm SD from three independent experiments $\left({ }^{* *} p<0.01 \mathrm{vs}\right.$. control)

'probable metabolite transport protein', and so forth. Among them, more than $75 \%$ DEGs which are associated with intracellular hypocrellin biosynthesis were up-regulated compared with control group (Additional file 2: Table S5 and Fig. 4a), such as laccase-like multicopper oxidase (MCO, TRINITY_DN31904_c0_ g1_i4), conidial yellow pigment biosynthesis polyketide synthase (PKS, TRINITY_DN73250_c0_g1_i1), hydroxyindole-O-methyltransferase (Omef, TRINITY DN28714_c0_g2_i1), FAD dependent oxidoreductase (FAD, TRINITY_DN33725_c0_g1_i8), phenol hydroxylase (Hyd, TRINITY_DN84020_c0_g1_i1), cytochrome P450 CYP2 subfamily (Mono, TRINITY_DN31508_c0_ g2_i1), fasciclin (Fas, TRINITY_DN43991_c0_g1_i1) (Table 2). The expression levels of seven randomly selected unigenes related to hypocrellin biosynthesis were confirmed through qRT-PCR strictly (Fig. 4b), in which $H y d$ and PKS were more dramatically up-regulated by 59.35 - and 14.59-fold, separately. The expression changes by qRT-PCR showed a similar tendency to those in transcriptome data (Table 2). In addition, it was found that more than $60 \%$ DEGs bound up with extracellular HA exportation were also up-regulated (Additional file 2: Table S6), such as major facilitator superfamily (MFS, TRINITY_DN33647_c1_g1_i8), $\mathrm{ABC}$ superfamily $(A B C$, TRINITY_DN73211_C0_g2 i1), secondary metabolites biosynthesis, transport and catabolism (MTP, TRINITY_DN30127_c0_g1_i1) (Table 2). Meanwhile, the SNP-induced up-regulation of transcriptional expression of selected unnigene $M F S$ (TRINITY_DN33647_c1_g1_i8) from 2.40- to 7.31-fold was confirmed by qRT-PCR (Fig. 4c).

As shown in Additional file 2: Table S7, a total of 6440 unigenes were classified into 55 transcription factor (TF) groups, including $\mathrm{C} 2 \mathrm{H} 2$, Trihelix, bZIP, bHLH, MYB related and so forth. Among all the TFs identified, bHLH (12.61\%), ERF (7.47\%), and MYB-related TF $(7.08 \%)$ were of a higher proportion. Furthermore, there were altogether 189 DEGs enriched into 27 TFs differently expressed under SNP treatment (Additional file 2: Table S8), in which 24 TFs (110 DEGs) were upregulated while $20 \mathrm{TFs}$ (53 DEGs) were down-regulated 
(See figure on next page.)

Fig. 4 a Heat map of DEGs involved in hypocrellin biosynthesis of Shiraia sp. S9 by SNP. b Validation of the expression levels of unigenes related to hypocrellin biosynthesis of S9 strain by qRT-PCR on day 8. Hyd, TRINITY_DN84020_c0_g1_i1.PKS, TRINITY_DN73347_C0_g1_i1.MCO, TRINITY_ DN31904_C0_g1_i4. FAD, TRINITY_DN33725_c0_g1_i8. Omef, TRINITY_DN28714_c0_g2_i1. Mono, TRINITY_DN33456_c1_g1_i4. Fas, TRINITY_ DN43991_C0_g1_i1. c Validation of the expression levels of MFS (TRINITY_DN33647_c1_g1_i8) by qRT-PCR. The SNP treatment was the same as specified in Fig. 3. Values are mean \pm SD from three independent experiments (** $p<0.01 \mathrm{vs}$. control)

in Shiraia mycelia. Among the up-regulated TFs, ERF ranked the highest (12 DEGs), followed by $\mathrm{C} 3 \mathrm{H}(12$ DEGs), and MYB (11 DEGs) (Table 2). Meanwhile, in the down-regulated TFs, NAC ranked the highest (8 DEGs), followed by C2H2 (6 DEGs), and bHLH (5 DEGs). Some prominent expression changes of unigenes associated with the high proportion of TFs were listed in Table 2, including several hypothetical proteins (TRINITY_DN31904_c0_g1_i4, TRINITY_ DN33640_c0_g1_i50, TRINITY_DN33640_c0_g1_i25 and TRINITY_DN28413_c0_g1_i2), two aldehyde dehydrogenases (TRINITY_DN33276_c0_g1_i17 and TRINITY_DN33276_c0_g1_i28), hexose transporterlike protein (TRINITY_DN31580_c0_g1_i1), ATP binding (TRINITY_DN31301_c0_g1_i7).

\section{SNP-induced transcriptional changes of genes involved in membrane permeabilization}

On the basis of the analysis results from GO classification (Additional file 2: Table S4), a large number of unigenes were enriched into 'membrane' (61 DEGs of GO:0016020), 'transporter' (51 DEGs of GO:0006810), 'integral to membrane' (36 DEGs of GO:0016021), 'intrinsic to membrane' (36 DEGs of GO:0031224), 'membrane part' (36 DEGs of GO:0,044,425), 'transmembrane transport' (34 DEGs of GO:0055085), 'transporter activity' (33 DEGs of GO:0005215) and 'transmembrane transporter activity' (30 DEGs of GO:0022857) items after SNP application. Hence, we explored the alteration of hyphal cell membrane permeability and membrane lipid components of Shiraia sp. S9. As shown in Fig. 5, the fluorescence was strengthened in SYTOX Green-stained cells after SNP treatment, indicating the increased permeabilization of cell membrane. Simultaneously, we found that the composition proportions of some saturated fatty acids such as palmitic (C16:0) and stearic (C18:0) were decreased by $19.08 \%$ and $23.97 \%$, respectively (Table 3 ). On the contrary, the proportions of four unsaturated fatty acids including palmitoleic (C16:1), oleic (C18:1), erucic (C22:1) and nervonic (C24:1) were raised significantly, up to 6.23-fold (Table 3). Hence, the ratio of unsaturated fatty acids with saturated ones was enhanced to 0.57 in the hyphal cells of S9 strain under SNP treatment, a 4.07fold of the control group, suggesting the enhancement of cell membrane fluidity.

\section{SNP-induced transcriptional changes of genes involved in oxidative stress}

According to the transcriptome analysis, there were 121 DEGs enriched into 'oxidation-reduction process' (GO:0055114), 'oxidoreductase activity' (GO:0016491), 'electron carrier activity' (GO:0009055) and 'oxidoreductase activity, acting on paired donors, with incorporation or reduction of molecular oxygen' (GO:0016705) items (Additional file 2: Table S4), suggesting an oxidative stress induced by SNP. By comparison between control and SNP groups (Fig. 6a), the green fluorescent signals of 2, 7-dichlorodihydroflurescein diacetate (DCFH-DA) in hyphal cells were brighter and more intense (4.72-fold, Fig. 6b), indicating the increase of ROS generation. When Shiraia sp. S9 was cultured in the presence of ROS scavenger vitamin C (Vc) and diphenyleneiodonium (DPI), an inhibitor of ROS generating enzyme NADPH oxidase (NOX) for $30 \mathrm{~min}$ prior to SNP application, the relative intensities of fluorescence in mycelia exhibited much more notable reduction of $72.39 \%$ and $72.14 \%$ (Fig. 6b) compared with the SNP group, separately. The content of superoxide anion $\left(\mathrm{O}_{2}{ }^{-}\right)$in mycelia was induced rapidly around $30 \mathrm{~min}$ of SNP treatment, reaching a highest value of $6.47 \mu \mathrm{mol} / \mathrm{g}$ FW (fresh weight) with time up to day 7, which was $59.14 \%$ higher than that of control (Fig. 6c). The change trends of hydrogen peroxide $\left(\mathrm{H}_{2} \mathrm{O}_{2}\right)$ concentration were consistent with $\mathrm{O}_{2}^{-}$production. The generation of $\mathrm{H}_{2} \mathrm{O}_{2}$ was strikingly increased from 9.12 to $11.75 \mu \mathrm{mol} / \mathrm{g}$ FW after 2-4-day treatment of SNP and then decreased on day 7-9, but it was still higher than those of the control group (Fig. 6d). As shown in Fig. 7, the activities and expression levels of three oxidoreductases were significantly stimulated by SNP. The most significant stimulation of enzyme activities of NOX, catalase (CAT) and superoxide dismutase (SOD) occurred on day 7 , which were $157.71 \%, 85.49 \%$ and $64.71 \%$ higher than those of control, respectively. Accordingly, the transcriptional expression levels of $N O X, C A T$ and $S O D$ were activated on day 5 or 7 , and the strongest induction effect appeared on day 7 , about 3.71-, 2.90- and 4.21-fold of control, separately.

\section{Discussion}

SNP is a potent vasodilator used clinically to treat hypertensive emergencies and heart failure. In addition, it is widely used as nitric oxide donor in pharmacologic studies to investigate on the physiological roles of NO [14]. In plant cells or root cultures, SNP has been used as NO 
$\mathbf{a}$<smiles>C#CC1CCCCC1</smiles>
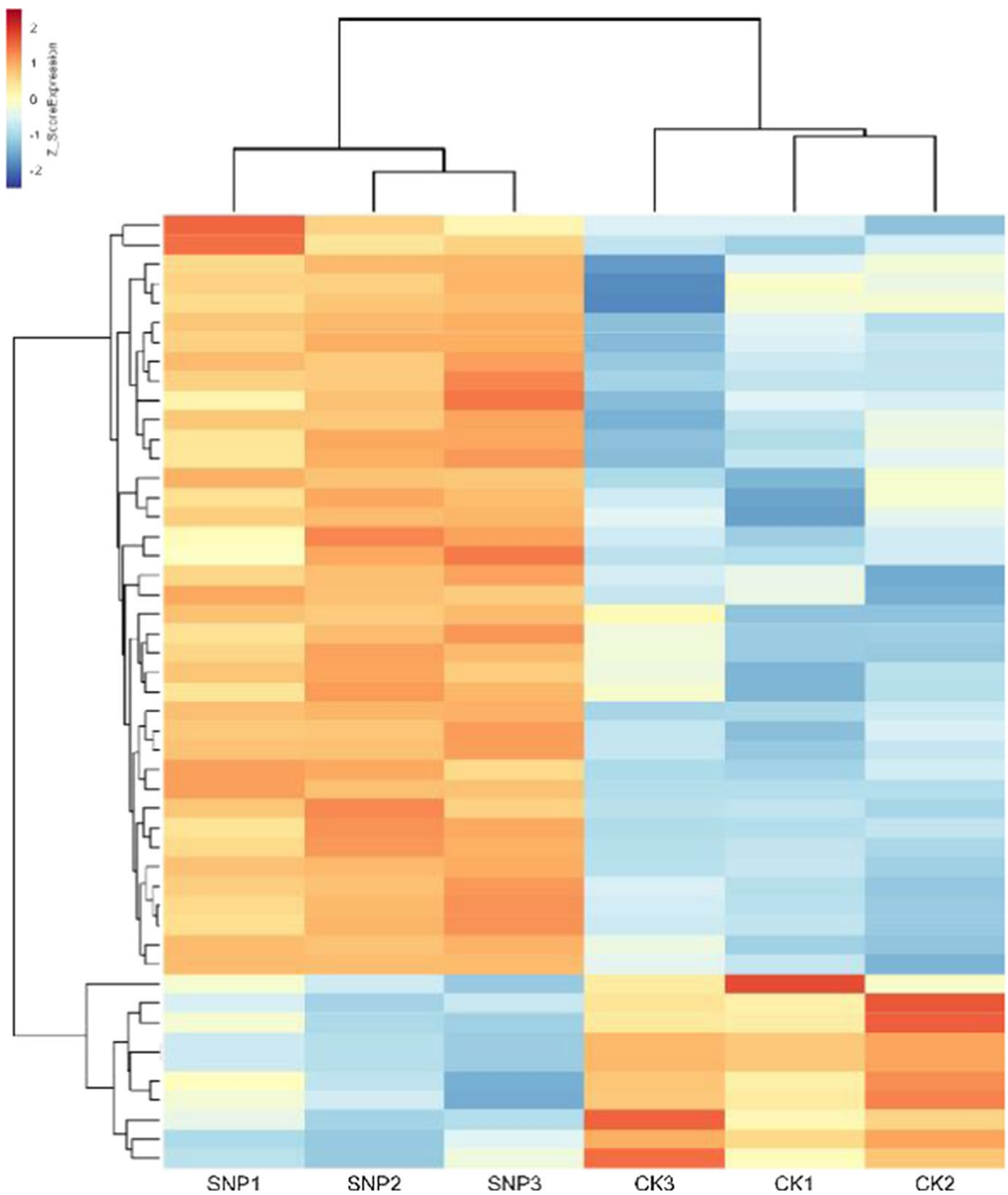

TRINITY DN32187 c0 g1 i8 TRINITY DN31232- $\mathrm{CO}^{-\mathrm{g} 1}$ - i6 TRINITY DN31904_C0 91 i4 TRINITY-DN27723-C0-g1-i1 TRINITYDN $31904^{-} \mathrm{CO}^{-} \mathrm{g} 1$ - 2 TRINITY DN32843 C0 g1-i1 TRINITYDN73347 CO 91 TRINITY DN32346 CO 91 TRINITY-DN28714- ${ }^{-}{ }^{-}-11$ TRINITY-DN44564- $\mathrm{CO}^{-} \mathrm{g}^{-}-1$ TRINITY-DN27083- $0^{-92}$ TRINITY DN53311 CO TRINITY-DN33456 C1 G1-14 TRINITY DN33456 1 - 110 TRINITY-DN84020-CO-91 TRINITY-DN33274-CO-917

TRINITY DN73250 C0-91-1

TRINITY-DN31010-CO-g1-

TRINY-DN3181-CO-g1-

TRNIY DN3

TRINIY-DN32187

TRINY DN12587-

TRINY-DN3418-0 TRITY-ON4500 RRIN D 4

TRNY-DN320

RRIN_DN3214-c0-g1

R N D D

TRNY DN64473 CO_G1

RRIN_DN33725_c0_g1_i8

TRINY-DN65064_Co-g1

TRINY_DN43991_c0_g1_1

RRINY DN44228 cog1

TRNAYDN27065 C0_91 i1

TRINITY_DN33332_c1_g1_18

TRRITY DN31811 c0 g1 is

TRINITY_DN12587 C0 g1 12

TRINITY-DN32187 $\mathrm{CO}^{-\mathrm{g} 1}$

TRINITY DN32346 c0 91 i6

RINITY DN44863 c0 g1 i1

RINITY DN44097 c0 ${ }^{-1}$ i1 TRINITY DN26074 CO 22 TRINITY DN33456 c1 g1 i 3 TRINITY DN33456 c1 g1 $^{-139}$ TRINITY DN55358 c0 $\mathrm{g} 1$ i1 TRINITY DN28767 CO $\mathrm{g} 3 \mathrm{i}$

TRINITY DN28354 C0 11

TRINITY DN28415 C0

TRINITY DN52967 C0 g2 i1

CK1

CK2
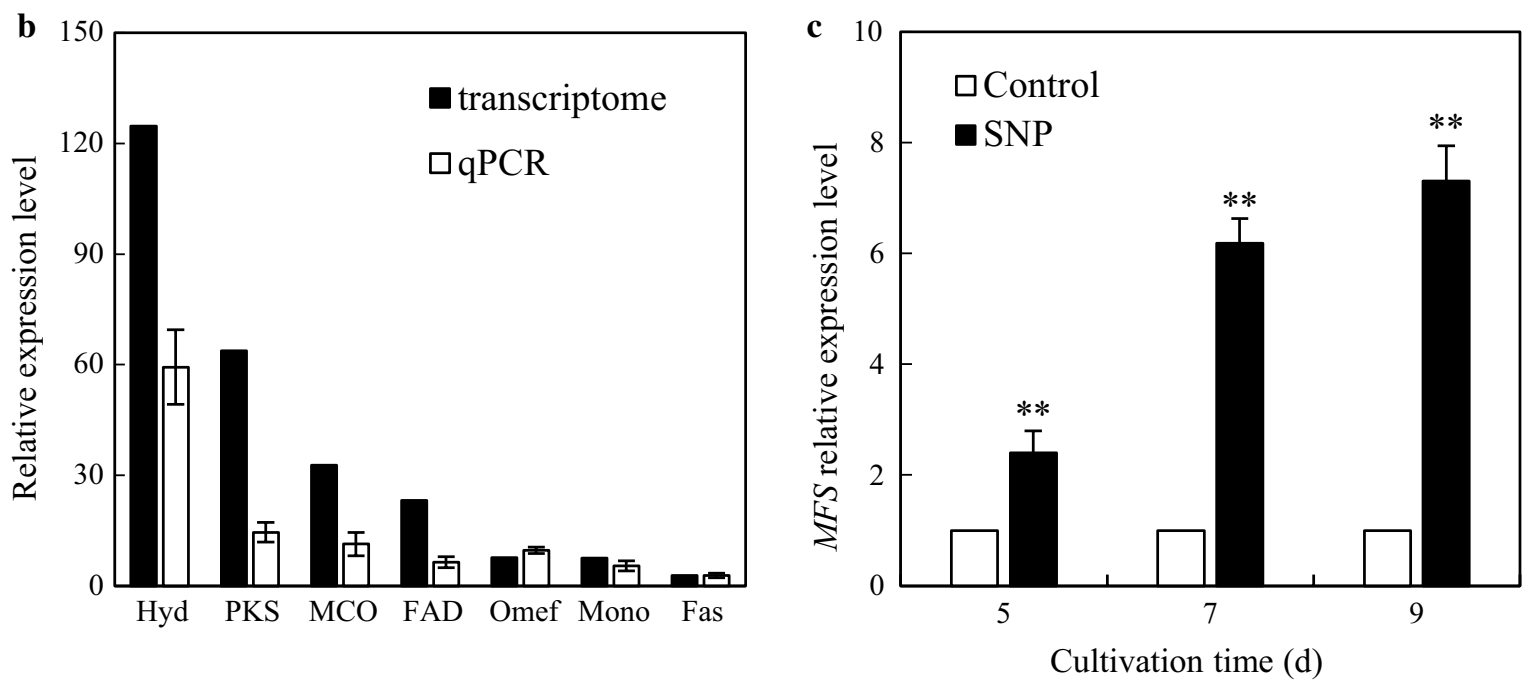
Table 2 Examples of DEGs involved in the hypocrellin biosynthesis, transport and transcription factors (TFs) of Shiraia sp. S9 by SNP

\begin{tabular}{|c|c|c|c|}
\hline Unigene ID & Up/down & Fold change $^{a}$ & Description \\
\hline \multicolumn{4}{|l|}{ Hypocrellin biosynthesis } \\
\hline \multicolumn{4}{|c|}{ 1. Laccase-like multicopper oxidase (MCO) } \\
\hline TRINITY_DN31904_C0_g1_i4 & Up & 32.8 & Multicopper oxidases [KOG1263] \\
\hline TRINITY_DN31904_C0_g1_i2 & Up & 21.18 & Multicopper oxidases [KOG1263] \\
\hline \multicolumn{4}{|l|}{ 2. Polyketide synthase (PKS) } \\
\hline TRINITY_DN73347_c0_g1_i1 & Up & 63.84 & Iterative polyketide synthase CazM [AOAOKOMCJ4.1] \\
\hline TRINITY_DN73250_C0_g1_i1 & Up & 7.18 & Conidial yellow pigment biosynthesis polyketide synthase [Q03149.2] \\
\hline \multicolumn{4}{|l|}{ 3. O-methyltransferase (Omef) } \\
\hline TRINITY_DN28714_C0_g2_i1 & Up & 7.74 & $\begin{array}{l}\text { Hydroxyindole-O-methyltransferase and related SAM-dependent methyltransferases } \\
\text { [KOG3178] }\end{array}$ \\
\hline TRINITY_DN32187_C0_g1_i3 & Up & 4.49 & $\begin{array}{l}\text { Hydroxyindole-O-methyltransferase and related SAM-dependent methyltransferases } \\
\text { [KOG3178] }\end{array}$ \\
\hline \multicolumn{4}{|c|}{ 4. FAD/FMN-dependent oxidoreductase (FAD) } \\
\hline TRINITY_DN33725_c0_g1_i8 & Up & 23.27 & FAD dependent oxidoreductase [OAL49443.1] \\
\hline TRINITY_DN64473_C0_g1_i1 & Up & 4.7 & Uncharacterized FAD-linked oxidoreductase ARB_02478 [D4AS41.2] \\
\hline \multicolumn{4}{|l|}{ 5. Hydroxylase $(H y d)$} \\
\hline TRINITY_DN84020_c0_g1_i1 & Up & 124.71 & Phenol hydroxylase [KMK58601.1] \\
\hline TRINITY_DN13072_C0_g1_i1 & Up & 18.1 & Alkane hydroxylase 1 [Q9Y757.2] \\
\hline \multicolumn{4}{|l|}{ 6. Monooxygenase (Mono) } \\
\hline TRINITY_DN25739_c0_g1_i1 & Up & 20.83 & FAD-dependent monooxygenase andF [G3Y424.1] \\
\hline TRINITY_DN31508_C0_g2_i1 & Up & 15.56 & Cytochrome P450 CYP2 subfamily [KOG0156] \\
\hline \multicolumn{4}{|l|}{ 7. Fasciclin (Fas) } \\
\hline TRINITY_DN43991_C0_g1_i1 & Up & 2.93 & Fasciclin and related adhesion glycoproteins [KOG1437] \\
\hline \multicolumn{4}{|l|}{ Hypocrellin transport } \\
\hline \multicolumn{4}{|l|}{ 1. Major facilitator superfamily (MFS) } \\
\hline TRINITY_DN33647_c1_g1_i8 & Up & 43.12 & Major facilitator superfamily [KOG0255] \\
\hline TRINITY_DN6606_C0_g1_i1 & Up & 37.62 & MFS quinate transporter-like protein QutD [OAL06848.1] \\
\hline \multicolumn{4}{|c|}{ 2. ATP-binding cassette transporter ( $A B C)$} \\
\hline TRINITY_DN73211_C0_g2_i1 & Up & 2.60 & ABC superfamily [KOG0065] \\
\hline TRINITY_DN30218_C0_g1_i2 & Down & 2.47 & ABC superfamily [KOG0055] \\
\hline \multicolumn{4}{|l|}{ 3. Metabolite transport protein (MTP) } \\
\hline TRINITY_DN30127_c0_g1_i1 & Up & 4.02 & Secondary metabolites biosynthesis, transport and catabolism [KOG0222] \\
\hline TRINITY_DN25695_c4_g2_i1 & Up & 2.23 & Secondary metabolites biosynthesis, transport and catabolism [KOG1208] \\
\hline \multicolumn{4}{|l|}{ Transcription factors (TFs) } \\
\hline \multicolumn{4}{|l|}{ 1. ERF } \\
\hline TRINITY_DN31904_C0_g1_i4 & Up & 5.04 & Hypothetical protein SNOG_06494 [XP_001796864.1] \\
\hline TRINITY_DN31580_C0_g1_i1 & Up & 4.67 & Hexose transporter-like protein [OAK96729.1] \\
\hline \multicolumn{4}{|l|}{ 2. $\mathrm{C} 3 \mathrm{H}$} \\
\hline TRINITY_DN33456_c0_g1_i1 & Up & 2.36 & P-loop containing nucleoside triphosphate hydrolase protein [OAG15388.1] \\
\hline TRINITY_DN31301_C0_g1_i7 & Up & 2.02 & ATP binding [KZM25621.1] \\
\hline \multicolumn{4}{|l|}{ 3. MYB } \\
\hline TRINITY_DN33687_C0_g1_i33 & Up & 7.01 & U4/U6-associated splicing factor PRP4 [KOG0670] \\
\hline TRINITY_DN33611_c1_g2_i5 & Up & 6.67 & ATP-dependent RNA helicase MSS116 [OAL02684.1] \\
\hline \multicolumn{4}{|l|}{ 4. NAC } \\
\hline TRINITY_DN33640_c0_g1_i50 & Down & 8.87 & Hypothetical protein IQ06DRAFT_327513 [OAK97976.1] \\
\hline TRINITY_DN33640_c0_g1_i25 & Down & 8.48 & Hypothetical protein SNOG_15601 [XP_001805746.1] \\
\hline \multicolumn{4}{|l|}{ 5. $\mathrm{C} 2 \mathrm{H} 2$} \\
\hline TRINITY_DN28764_C0_g1_i5 & Down & 2.74 & Acetyl-CoA synthetase-like protein [OAL06367.1] \\
\hline TRINITY_DN28413_CO_g1_i2 & Down & 2.61 & Hypothetical protein SNOG_13514 [XP_001803722.1] \\
\hline \multicolumn{4}{|l|}{ 6. bHLH } \\
\hline TRINITY_DN33276_C0_g1_i17 & Down & 7.94 & Aldehyde dehydrogenase [OAK94834.1] \\
\hline TRINITY_DN33276_C0_g1_i28 & Down & 6.32 & Aldehyde dehydrogenase [OAK94834.1] \\
\hline
\end{tabular}


Table 2 (continued)

${ }^{a}$ Fold change, up: ratio (S2/S1); down: ratio (S1/S2). S1, the FPKM value of the unigene in control group; S2, the FPKM value of the unigene in SNP group

Table 3 Effect of SNP treatment on fatty acid composition (\% total fatty acid) of Shiraia sp. 59

\begin{tabular}{lll}
\hline Fatty acid composition & \multicolumn{2}{l}{ Total fatty acid (\%) } \\
\cline { 2 - 3 } & Control & SNP \\
\hline C16:0 & $24.21 \pm 0.54$ & $19.59 \pm 1.07^{* *}$ \\
C18:0 & $15.02 \pm 0.35$ & $11.42 \pm 0.16^{* *}$ \\
C23:0 & $0.18 \pm 0.03$ & $0.28 \pm 0.05$ \\
C16:1 & $3.49 \pm 0.24$ & $8.28 \pm 0.21^{* *}$ \\
C18:1 & $1.17 \pm 0.10$ & $4.79 \pm 0.42^{* *}$ \\
C22:1 & $0.62 \pm 0.04$ & $3.86 \pm 0.57^{* *}$ \\
C24:1 & $0.29 \pm 0.03$ & $0.82 \pm 0.11^{* *}$ \\
Unsaturated/saturated fatty & $0.14 \pm 0.01$ & $0.57 \pm 0.01^{* *}$ \\
acid ratio & & \\
\hline
\end{tabular}

The SNP treatment was the same as specified in Fig. 3. Ratio of unsaturated/ saturated fatty acid $=(C 16: 1+\mathrm{C} 18: 1+\mathrm{C} 22: 1+\mathrm{C} 24: 1) /(\mathrm{C} 16: 0+\mathrm{C} 18: 0+\mathrm{C} 23: 0)$. Values are mean \pm SD from three independent experiments. ** $p<0.01$ vs. control

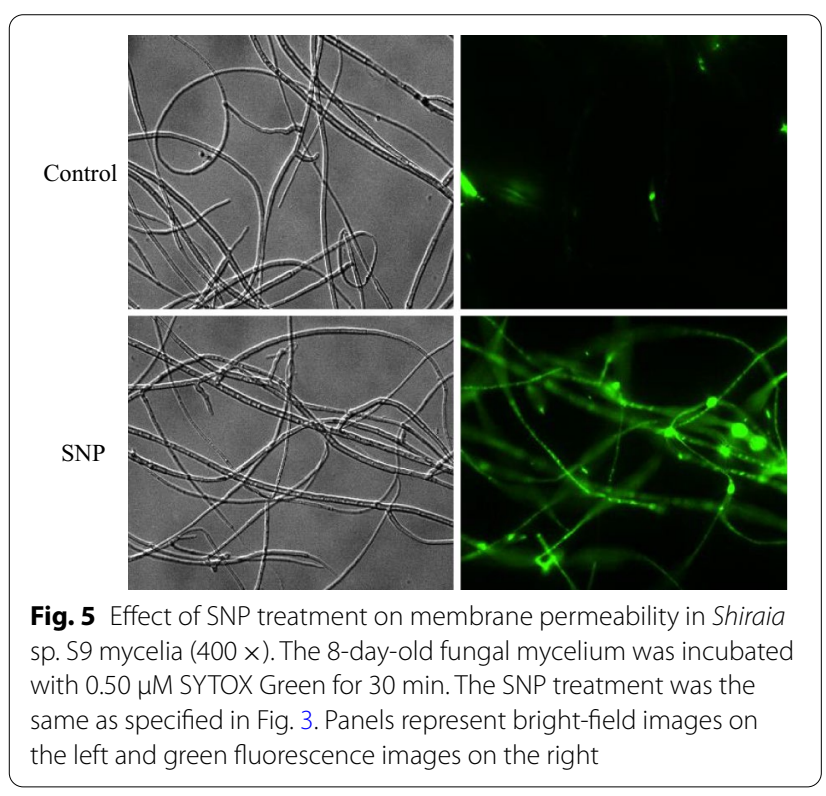

donor to elicit the production of important bioactive secondary metabolites such as anthocyanin and flavonol glycoside in Pisum sativum [30], and tropane alkaloids in hair roots culture of Hyoscyamus reticulatus [31]. More recently, the signal roles of NO in fungal developmental and metabolic biosynthesis have drawn more attention [32]. With the help of using NO donor SNP and NO scavengers, previous studies indicated that NO modulated the germination of $C$. coccodes conidia [21], hyphal elongation of Magnaporthe oryzae [33] and biosynthesis of fungal secondary metabolites such as styrylpyrone polyphenols, flavonoids and phenolics [23, 34]. However, it is less reported on SNP application as a chemical elicitor to enhance fungal metabolite production in mycelium cultures. In submerged cultures of G. lucidum, the eliciting effects of SNP at $0.5-5.0 \mathrm{mM}$ on ganoderic triterpenoid production were investigated over $120 \mathrm{~h}$ [24]. The yields of ganoderic triterpenoids were increased by $40.94 \%$ at $72 \mathrm{~h}$ after SNP treatment at $5 \mathrm{mM}$. In our present study, NO elicited the biosynthesis of hypocrellins in Shiraia sp. S9, especially increased EA and HA contents in the mycelia (Table 1 and Additional file 1: Figure S3). After the optimization of the conditions for SNP application (the concentration and adding time) (Additional file 1: Figure S4 and S5), a higher production of HA $(110.34 \mathrm{mg} / \mathrm{L})$ was induced by SNP at $0.02 \mathrm{mM}$ on day 7 , a 2.65 -fold increase over the control (Fig. 3). Compared with other elicitors used to promote HA production in Shiraia cultures including light irradiation $[35,36]$, ultrasound exposure [25] or microbial elicitors [9, 37], the chemical elicitor SNP has the advantages of lower cost, easy preparation, no growth suppression, and high efficiency for eliciting metabolite production in the mycelium cultures.

In our study, a rapid generation of endogenous $\mathrm{NO}$ was observed when SNP was applied (Fig. 1). The generated $\mathrm{NO}$ in mycelia was fully suppressed by a NO scavenger cPTIO, indicating exogenous SNP as a NO producer in the culture. Accumulating evidence showed NO could collaborate with the accompanied generation of ROS to form nitro-oxidative stress involved in human or plant diseases [14, 38]. Zheng et al. (2010) reported that the SNP $(50 \mu \mathrm{M})$ promoted the $\mathrm{H}_{2} \mathrm{O}_{2}$ content in the hairy roots of $A$. annua elicited by fungal oligosaccharides [39]. SNP at $250 \mu \mathrm{M}$ could enhance $\mathrm{H}_{2} \mathrm{O}_{2}$ level in adventitious roots of E. purpurea [17]. In this study, SNP treatment also resulted in an accumulation of ROS including $\mathrm{O}_{2}{ }^{-}$and $\mathrm{H}_{2} \mathrm{O}_{2}$ in the mycelia (Fig. 6). Simultaneously, $\mathrm{GO}$ analysis demonstrated the enriched DEGs of 'oxidation-reduction process' (GO:0055114), 'oxidoreductase activity' (GO:0016491) and 'electron carrier activity' (GO:0009055) in the group of molecular function (Additional file 1: Figure S8 and Additional file 2: Table S4). Both the gene expressions of ROS generating NOX and the activities of antioxidant enzymes such as CAT and SOD were validated in SNP-treated mycelia (Fig. 7), suggesting that SNP could activate NOX for the increased oxidative stress in the mycelium cultures. In fungal 

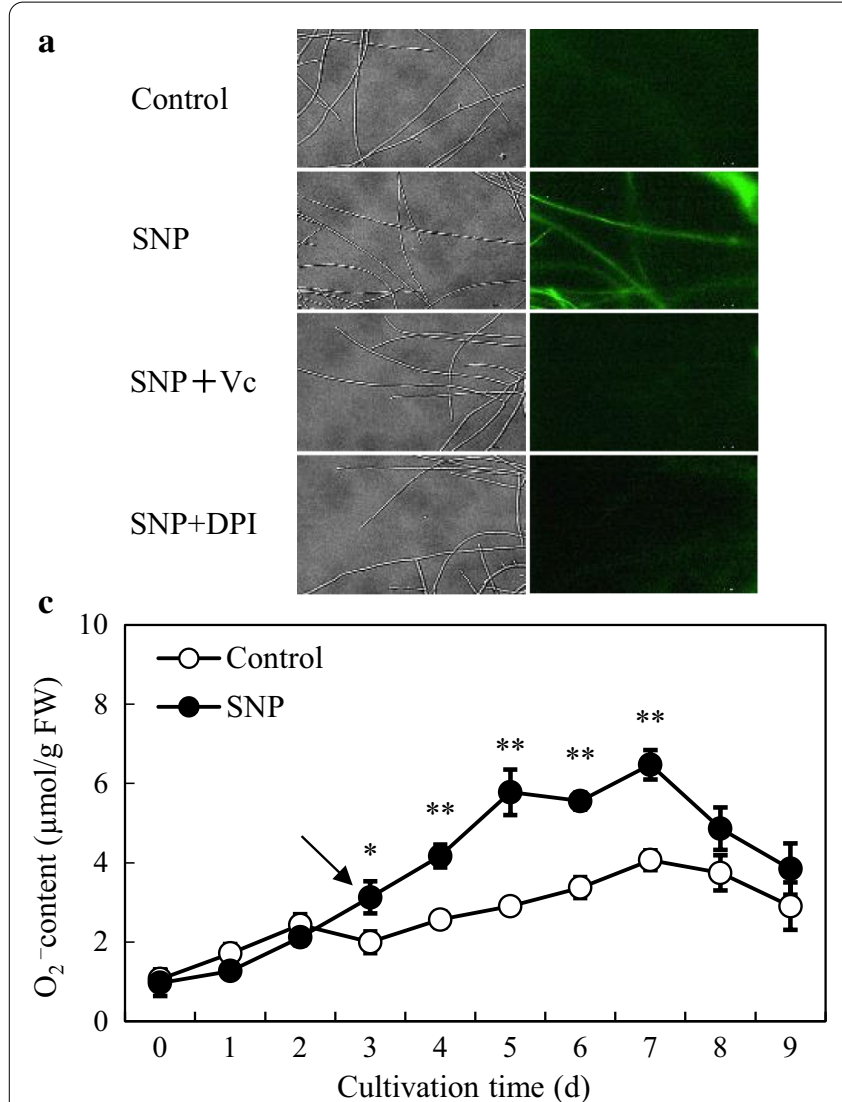

b
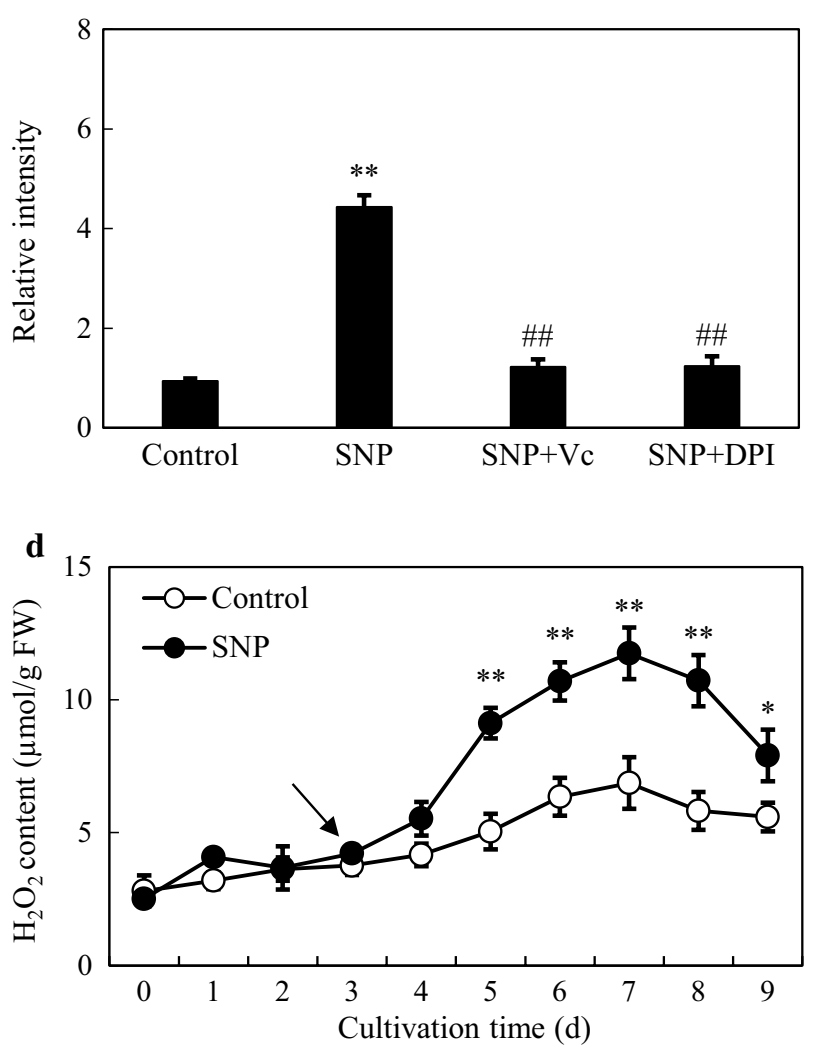

Fig. 6 Effect of SNP treatment on ROS production in mycelium of Shiraia sp. S9. a Bright-field images (left) and fluorescence microscopy (right) of DCFH-DA-stained mycelia $(400 \times)$. b ROS accumulation in mycelium after SNP treatment. Vc $(0.01 \mathrm{mM})$ and DPI (5.00 $\mu \mathrm{M})$ were added 30 min prior to SNP treatment. The contents of $\mathrm{O}_{2}^{-} \mathbf{c}$ and $\mathrm{H}_{2} \mathrm{O}_{2} \mathbf{d}$ in mycelium of $\mathrm{S} 9$ by SNP. The photos and contents of ROS were taken and detected after $2 \mathrm{~h}$ of SNP treatment. The SNP treatment was the same as specified in Fig. 3. The arrow represents the time of SNP addition. Values are mean \pm SD from three independent experiments $\left({ }^{*} p<0.05,{ }^{* *} p<0.01\right.$ vs. control and ${ }^{\# \#} p<0.01$ vs. SNP)

cultures, ROS could provoke the production of fungal secondary metabolites, such as carotenoid in Neurospora crassa [40], deoxynivalenol in Fusarium graminearum [41], ochratoxin A in A. ochraceus [42]. In our previous researches, ROS generation and oxidative stress were reported as early signal-response events leading to HA biosynthesis of Shiraia under various abiotic elicitors, including the applications of surfactant Triton X-100 [10], lower intensity ultrasound [25] and light-dark shift [35]. These studies indicated the oxidative stress was involved in the hypocrellin biosynthesis of Shiraia. In our present study, we found 54 putative unigenes from 571 DEGs probably involved in hypocrellin biosynthesis after SNP treatment (Additional file 2: Table S5 and Fig. 4a). It was supposed that hypocrellins were biosynthesized in fungal cells via repetitive decarboxylative condensation of acetyl- and malonyl-CoA by polyketide synthase $(P K S)$, methylation, decarboxylation and reduction by O-methyltransferase (Omef), monooxygenase/hydroxylase $(M o n o / H y d)$ and multicopper oxidase $(M C O)$, and then the core structure formation via the cooperation of fasciclin (Fas), laccase and berberine bridge enzyme $(B B E)[28,43,44]$. The deletion and overexpression of SbaPKS indicated its essential role in the hypocrellin biosynthesis of Shiraia sp. SUPER-H168 [45]. SbaPKS also had a moderating effect on the transcriptional expression of its adjacent genes FAD, Omef, Mono and MCO in the gene cluster. Overexpression of Hyd gene in $S$. bambusicola S4201 resulted in ultra-higher production of HA (1180.1 mg/L) [46]. Most of above-mentioned unigenes have been up-regulated significantly in our present experiment after SNP application, especially the expression of Hyd (59.35-fold) and PKS (14.59-fold) genes (Fig. 4b). The first transcription factor involved in HA biosynthesis is the zinc finger transcription factor $\mathrm{ztf}$ [29]. The $z f t f$ gene with a GAL4-like Zn(II)2Cys6 (or C6 zinc) binuclear cluster DNA-binding domain is located in the hypocrellin gene cluster. However, $z \mathrm{ftf}$ is not the differentially expressed gene induced by SNP treatment (Additional file 2: Table S8). The up-regulated TFs were 
$\mathbf{a}$

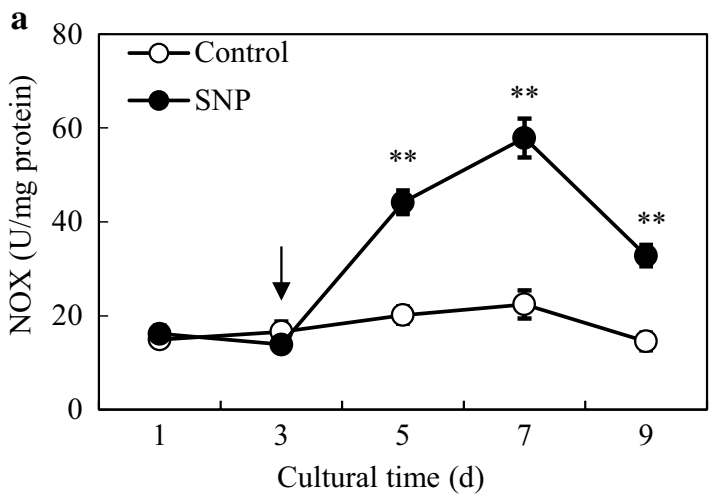

c
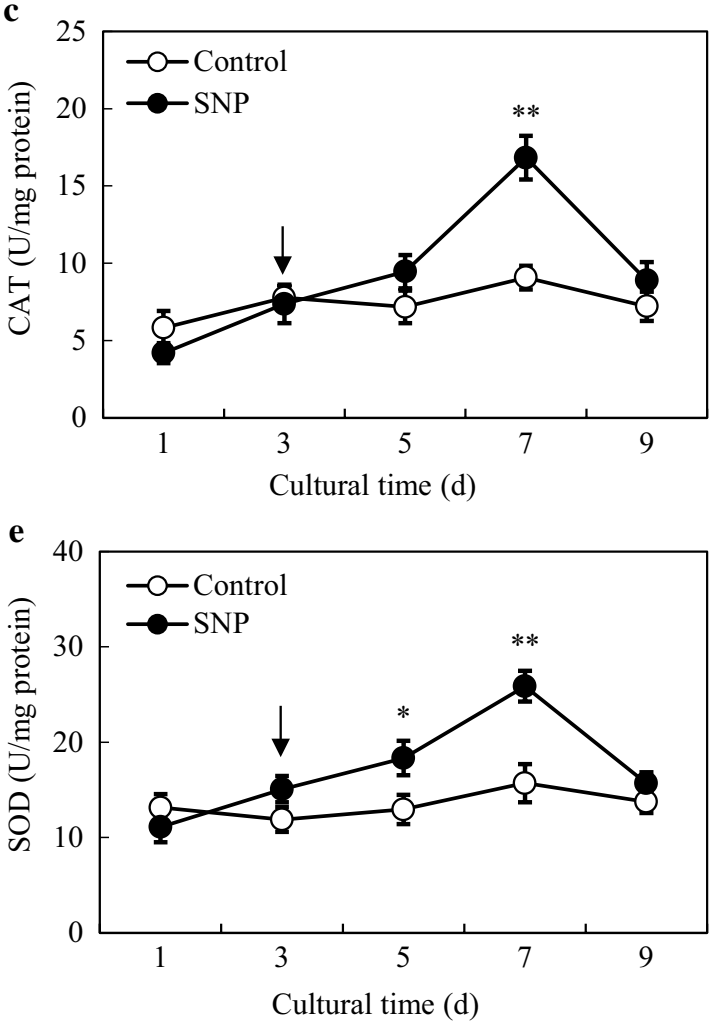

.

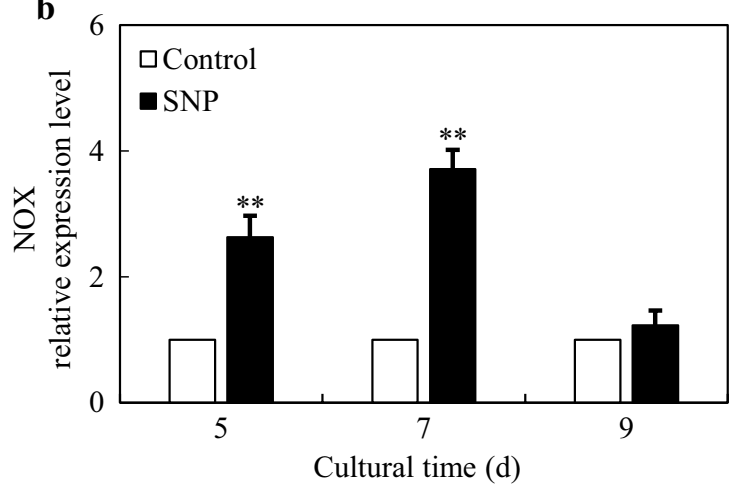

d
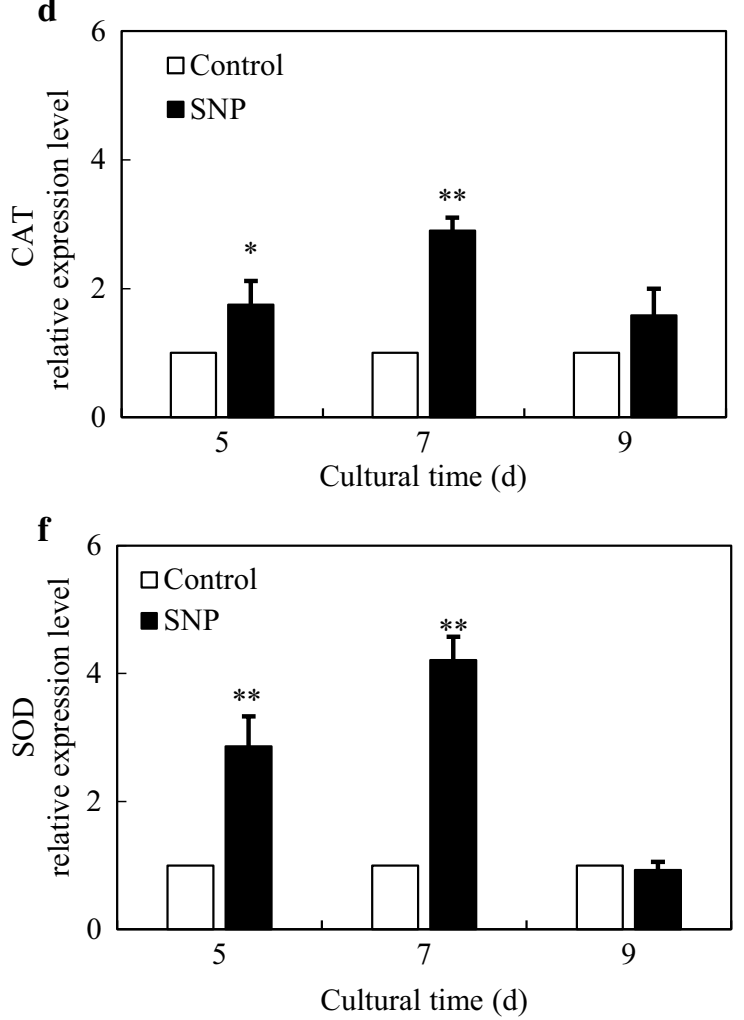

Fig. 7 Effects of SNP treatment on activities and expressions of NOX (a, b), CAT (c, d) and SOD (e, f) in Shiraia sp. S9 mycelium on day 1-9. NOX (comp2367_c0_seq1), CAT (comp15524_c0_seq1), SOD (CL8477Contig1). The SNP treatment was the same as specified in Fig. 3. The arrow represents the time of SNP addition. Values are mean \pm SD from three independent experiments $\left({ }^{*} p<0.05\right.$ and ${ }^{* *} p<0.01$ vs. control)

detected in the present study, including ERF, C3H and MYB (Table 2). These TFs have been studied extensively in initiating plant stress responses through involvement in the biosynthesis of secondary metabolites such as terpenoid indole alkaloids, taxol, flavonoids and proanthocynanins [47]. The challenge would be to elucidate the regulatory role of these TFs in regulation of HA biosynthesis. Taken together, the results from our present study have shown that SNP induced oxidative stress and activated genes and TFs for hypocrellin biosynthesis. The relationship between $\mathrm{NO}$ and ROS, and the mechanism of their mediation on hypocrellin biosynthesis are being further investigated.

It is worth noting that SNP could promote hypocrellin both inside the hyphae and from the medium (Fig. 3). In mycelium cultures after SNP application, the released $\mathrm{HA}$ in culture medium was increased by $26.09-119.26 \%$ (Fig. 3c). Although the increased extracellular HA accounts for only $5.26-6.83 \%$ of total HA production, the results still implicated the efflux 
of hypocrellins induced by SNP. Our GO classification revealed that the enriched DEGs were mainly in the categories of 'membrane' (GO:0016020), 'integral to membrane' (GO:0016021), 'transmembrane transport' (GO:0055085) and 'transporter activity' after SNP application (Additional file 1: Figure S8 and Additional file 2: Table S4). SNP treatment increased the proportion of total unsaturated fatty acids in the membranes of fungal hyphae (Table 3), leading to the enhanced membrane permeability (Fig. 5). On the other hand, membrane transporters have been reported to convey a photoactivated toxin cercosporin from Cercospora fungi for the auto-resistance [48]. Our RNA-Seq data also displayed that 32 unigenes encoding the transporter protein major facilitator superfamily $(M F S)$ were up-regulated significantly (Additional file 2: Table S6). The qRT-PCR results also validated that the increases of gene expression levels of MFS (unigne No. TRINITY_DN33647_c1_g1_i8) (2.40- to 7.31-folds) after SNP application (Fig. 4b). These results indicated that SNP could induce changes of membrane permeability and activate membrane transporter such as MFS to facilitate the secretion of hypocrellins.

\section{Conclusion}

Although endogenous NO generation was investigated in Shiraia mycelium cultures under the treatment of fungal elicitors $[8,12]$, the regulation on fungal metabolites by NO has not been well studied. To the best of our knowledge, it is the first time to use SNP as a chemical elicitor in mycelium cultures to promote hypocrellin productions. SNP at a higher concentration $(0.02 \mathrm{mM})$ acted as a pro-oxidant, thereby raising the ROS generation, up-regulating biosynthetical genes for hypocrellin production. The increased membrane permeability and gene expression of member transporters induced by SNP was associated with efflux of hypocrellins. Our results showed the increase of total hypocrellin production is mainly attributed to the cellular hypocrellin biosynthesis and the efflux. When the mycelium cultures were treated by SNP at $0.02 \mathrm{mM}$ for 4 days, the highest production $(110.34 \mathrm{mg} / \mathrm{L})$ of HA was achieved without any fungal growth depression. Although the mechanism of the SNP elicitation needs further investigation, the present study successfully provided a novel chemical elicitor for the biotechnological production of hypocrellins. This study is useful for hypocrellin production in a large-scale culture of Shiraia and facilitates the exploring of unknown genes and novel fungal secondary metabolites induced by SNP.

\section{Materials and methods}

\section{Strain, media and culture conditions}

The strain Shiraia sp. S9 (CGMCC16369) for hypocrellin production was obtained from fresh Shiraia fruit-body in our previous work [49]. The strain was maintained and stored at $4{ }^{\circ} \mathrm{C}$ on a potato dextrose agar (PDA) slant and initially grown at $28{ }^{\circ} \mathrm{C}$ for 8 days. The details of medium components and culture conditions were provided by our previous report [25]. The seed broth $(2 \mathrm{~mL})$ of S9 was transferred into a 150-mL Erlenmeyer flask containing 50-mL liquid medium and maintained in a rotary shaker at $150 \mathrm{rpm}$ for 9 days at $28^{\circ} \mathrm{C}$.

\section{SNP application}

SNP (Beyotime Biotechnology, Jiangsu, China) was dissolved in deionized water at $0.50 \mathrm{mM}$ as a stock solution and filtered through a sieve $(\varnothing 0.22 \mu \mathrm{m})$ before use. To determine the optimal dosage of SNP for hypocrellin production, different dosages $(0.01-1.00 \mathrm{mM})$ of SNP were added into the cultural broth on day 3 . To confirm the effect of the addition time, $0.02 \mathrm{mM}$ SNP was added into the mycelium culture on day $1-5$ respectively.

\section{Measurement of medium $\mathrm{pH}$, residual glucose and $\mathrm{NO}$ accumulation}

The medium $\mathrm{pH}$ of the cultural broth was detected with pH Meter (FE20, Mettler Toledo, Zurich), and the residual glucose in cultural broth was measured using anthrone-sulfuric acid method [50]. To detect the NO accumulation in Shiraia sp. S9 mycelia, the NO-specific fluorescent probe DAF-2-DA (Sigma-Aldrich, St. Louis, MO, USA) was applied [51]. After 3 days of initial culture, DAF-2-DA $(0.50 \mu \mathrm{M})$ was added to the cultural broth prior to SNP $(0.10 \mathrm{mM})$ treatment. The NO scavenger cPTIO at $0.10 \mathrm{mM}$ was added $30 \mathrm{~min}$ prior to SNP treatment [13]. After $2 \mathrm{~h}$ of incubation, a fluorescent microscope (Olympus Cell'R IX81, Center Valley, PA, USA) with excitation/emission wavelengths $(470 \mathrm{~nm} / 525 \mathrm{~nm})$ was used for fluorescence observation.

\section{Observation of morphological characteristics}

To analyze the effect of SNP $(0.01-1.00 \mathrm{mM})$ on growth and morphological changes of Shiraia sp. S9 during the solid medium culture, the colony diameter was measured in triplicates ( 3 objects in a replicate). Photos were taken on day 6 . To investigate the influence of SNP on fungal development, the 8-day-old colony was eluted with $10-\mathrm{mL}$ deionized water. Subsequently, the fungal morphological characteristics were observed and photographed by a light microscope (CKX41, Olympus, Tokyo, Japan). To observe the influence of SNP on fungal growth during the submerged culture, SNP at $0.10 \mathrm{mM}$ was added into the cultural broth on day 3 . The mycelia were 
harvested and dried to constant weight at $60{ }^{\circ} \mathrm{C}$ to assess fungal biomass. The fungal pellets were viewed and photographed under a stereoscopic microscope (SMZ1000, Nikon, Tokyo, Japan). Pellet diameters were measured in triplicates (50 pellets per replicate).

\section{Analysis of cell membrane permeability}

The membrane permeabilization of hyphal cell was detected by SYTOX Green dye, a high-affinity nucleic acid stain fluorescent probe (Eugene, Oregeon, USA) [52]. After 5 days of the initial culture of $S 9$ strain, the harvested hyphae were incubated with the fluorescent probe solution $(0.50 \mu \mathrm{M})$ for $30 \mathrm{~min}$, and then the fluorescence intensity was observed and analyzed by means of an Olympus fluorescent microscope (CKX41, Tokyo, Japan) with the excitation/emission wavelengths at $488 / 538 \mathrm{~nm}$ respectively.

The components of mycelial fatty acids were extracted and analyzed via the protocols given in our previous report [10]. The detection of fungal fatty acids was carried out by using a gas chromatograph (Agilent7820, Palo Alto, CA, USA) with an Agilent DB-23 column (30.00 $\mathrm{m} \times 0.25 \mathrm{~mm}$ dimension). The fatty acids were quantified with their internal standards purchased from Sigma-Aldrich (St. Louis, MO, USA).

\section{Measurement of ROS accumulation and activities of antioxidant enzymes}

The ROS generation in hyphal cell was detected by DCFH-DA (Beyotime Biotechnology, Haimen, China) probe, which could penetrate the cell membrane freely [53]. After $2 \mathrm{~h}$ of SNP treatment, the harvested hyphae were incubated with the fluorescent dye solution $(0.01 \mathrm{mM})$ for $1 \mathrm{~h}$, and then the fluorescence intensity was observed and photographed by means of an Olympus fluorescent microscope (CKX41, Tokyo, Japan) with the excitation/emission wavelengths at $485 / 528 \mathrm{~nm}$. The relative fluorescence value is defined to the ratio of fluorescence intensity at 'SNP' group to that at 'Control' group. Simultaneously, the contents of $\mathrm{H}_{2} \mathrm{O}_{2}$ and $\mathrm{O}_{2}{ }^{-}$in the mycelia were assayed as previously described $[54,55]$. The activities of NOX, CAT and SOD were measured using Enzyme Activity Assay Kit (Beyotime Biotechnology, Nanjing, China) according to the manufacturer's protocol and previous reports [56-58].

To study the effects of ROS signal during the culture of S9 strain by SNP, Vc and DPI were used as ROS scavenger and NOX inhibitor, respectively. The concentrations of ROS scavenger and NOX inhibitor used in this work were chosen based on previous research [59]. Vc (0.01 mM) and DPI $(5.00 \mu \mathrm{M})$ were added to the culture at $30 \mathrm{~min}$ prior to the application of SNP $(0.02 \mathrm{mM})$ on day 3 . The relative fluorescence value is defined to the ratio of fluorescence intensity at 'SNP + Vc' or 'SNP + DPI' groups to that at 'SNP' group.

\section{Detection of hypocrellin production}

To analyze the influence of SNP on hypocrellin production in Shiraia sp. S9, SNP at $0.10 \mathrm{mM}$ was added on day 3 and cultivated for 8 days. The hypocrellins in fungal mycelium and cultural broth were extracted based on the previous report [60]. The individual hypocrellin was quantified using a reverse-phase Agilent 1260 HPLC system (Agilent Co., Wilmington, USA) equipped with $250.0 \mathrm{~mm} \times 4.6 \mathrm{~mm}$ Agilent HC-C18 column. Samples were eluted with a mobile phase of acetonitrile: water $(65: 35, \mathrm{v} / \mathrm{v})$ at $1 \mathrm{~mL} / \mathrm{min}$ for $20 \mathrm{~min}$ and monitored at $465 \mathrm{~nm}$. Individual hypocrellin was quantified separately with genuine standards provided by the Chinese National Compound Library (CNCL, Shanghai, China). Total hypocrellin production refers to the sum of intracellular and extracellular hypocrellin.

\section{Transcriptome sequencing, annotation and analysis}

In this study, six samples with or without SNP treatment were obtained from three biological replicates (independent experiments) to establish cDNA libraries. Then, the HiSeq X Ten platform (Illumina, San Diego, CA, USA) was applied to sequence above cDNA libraries. The raw reads were cleaned and assembled according to our previous report [61]. All unigenes were subsequently annotated based on Basic Local Alignment Search Tool (BLAST) searches (version 2.2.31+) against Gene Ontology (GO, http://www.geneontology.org/) database with a cut off $E$ value of $\leq 1 \mathrm{e}^{-5}$. The raw RNA-seq data have been submitted to NCBI's Gene Expression Omnibus (GEO) repository under accession number SRR72932007293205. In our study, we calculated the relative expression levels of genes via the fragments per kilobase per million reads (FPKM) method described by Mortazavi et al. [62]. Meanwhile, the significance of gene expression differences was evaluated using the cut-off criteria of $\mid$ foldchange $\mid \geq 2$ and $p$ value $<0.05$.

\section{Quantitative real-time polymerase chain reaction (qRT-PCR)}

Total RNA was extracted using RNAprep pure Plant Kit (Tiangen, Beijing, China) according to the instruction. The primer sequences of $18 \mathrm{~S}$ ribosomal RNA (internal reference gene) and selected unigenes from NCBI database of SRR7293200-7,293,205 and PRJNA323638 were listed in Additional file 1: Table S9. The qRT-PCR was performed according to the method in our previous study [61]. And, the transcriptional expression levels of selected genes were calculated from cycle threshold 
values by using the $2^{-\triangle \triangle \mathrm{CT}}$ method described in detail by Zhang et al. [63].

\section{Statistical analysis}

Data analyses were carried out using Microsoft Excel and expressed as Mean \pm Standard Deviation (SD). Student's $t$-test was applied for the comparison of the means between two groups. One-way analysis of variance (ANOVA) test was used for the comparison of the means among multiple groups. $p<0.05$ is considered statistically significant.

\section{Supplementary Information}

The online version contains supplementary material available at https://doi. org/10.1186/s12934-021-01581-8.

\section{Additional file 1: Table S1. Illumina RNA-Seq reads and de novo} assembly statistics of Shiraia sp. S9 by SNP. Table S2. Summary statistics of unigene annotation numbers of Shiraia sp. S9 by SNP. Table S9. Primers and relevant information of reference and target genes. F: forward primer, R: reverse primer. Figure S1. Effect of SNP at $0.10 \mathrm{mM}$ on the growth and development of Shiraia sp. 59 in submerged culture. Time profiles of fungal biomass (A) and average pellet diameters (B) during the production culture. Figure S2. Time profiles of $\mathrm{pH}(\mathrm{A})$ and residual sugar $(B)$ in Shiraia sp. S9 submerged cultures with the addition of SNP at $0.10 \mathrm{mM}$. Figure S3. Chromatograms of PQs in mycelium of Shiraia sp. S9 treated by SNP $(0.10 \mathrm{mM})$ on day 3 and cultivated for 8 days. Figure S4. Effect of SNP concentration on (A) hyphal growth, (B) HA content in mycelium and $(\mathrm{C})$ the released $\mathrm{HA}$ in cultural broth of Shiraia sp. S9. The strain was treated by SNP (0.01-1.00 mM) on day 3. Figure S5. Effect of SNP addition time on (A) hyphal growth, (B) HA content in mycelium and (C) the released HA in cultural broth of Shiraia sp. S9. SNP (0.02 mM) was added on different cultural time (1-5 d). Figure S6. The length distribution of unigenes of Shiraia sp. S9 by SNP. Figure S7. Heat map of DEGs summary of Shiraia sp. S9 by SNP. The up/down-regulated DEGs were detected by comparing RNA-Seq data of the unigenes of case group to control group (up: ratio > 1, down: ratio < 1). Figure S8. The cellular component $(C C)$, biological process (BP) and molecular function (MF) of gene ontology (GO) categories of the DEGs in Shiraia sp. S9 under SNP treatment at $0.02 \mathrm{mM}$. The arrow represents the time of SNP addition. Values are mean \pm SD from three independent experiments $\left({ }^{* *} p<0.01 \mathrm{vs}\right.$. control). Different letters above the bars mean significant differences $(p<0.05)$.

Additional file 2: Table S3. Annotation of DEGs using NR, SWISS-Prot KEGG, KOG and GO databases and their fold change with SNP treatment. Table S4. GO enrichment of Shiraia sp. S9 with SNP treatment. Table S5. Differentially expressed genes (DEGs) involved in hypocrellin biosynthesis of Shiraia sp. S9 under SNP treatment. Table S6. Differentially expressed genes (DEGs) involved in hypocrellin transport of Shiraia sp. S9 under SNP treatment. Table S7. Annotation of transcription factors in Shiraia sp. S9. Table S8. Differentially expressed genes (DEGs) involved in transcription factors of Shiraia sp. S9 under SNP treatment.

\section{Acknowledgements}

Not applicable.

\section{Authors' contributions}

JWW and YJM conceived the study and participated in its design. YW, XPL and YJM undertook experiments and data analysis. YJM, XPL and YW drafted the manuscript. JWW supervised the research and revised the paper. All authors read and approved the final manuscript.

\section{Funding}

This work was supported by the National Natural Science Foundation of China (Nos. 82073955 and 81773696) and the Priority Academic Program Development of the Jiangsu Higher Education Institutes (PAPD).

\section{Availability of data and materials}

All data generated or analyzed during this study are included in this published article and its Additional files.

\section{Declarations}

Ethics approval and consent to participate

Not applicable.

\section{Consent for publication}

Not applicable.

\section{Competing interests}

The authors declare that they have no competing interests.

\section{Author details}

${ }^{1}$ College of Pharmaceutical Sciences, Soochow University, Suzhou 215123, China. ${ }^{2}$ College of Life Sciences, Northwest Normal University, Lan-

zhou 730000, China.

Received: 15 December 2020 Accepted: 17 April 2021

Published online: 28 April 2021

\section{References}

1. Zhen J, Di W. Novel therapeutic and diagnostic applications of hypocrellins and hypericins. Photochem Photobiol. 1995;61:529-39.

2. Dai DQ, Phookamsak R, Wijayawardene NN, Li WJ, Bhat DJ, Xu JC, et al. Bambusicolous fungi. Fungal Divers. 2017;82:1-105.

3. Kishi T, Tahara S, Taniguchi N, Tsuda M, Tanaka C, Takahashi S. New perylenequinones from Shiraia bambusicola. Planta Med. 1991;57:376-9.

4. Mulrooey CA, O'Brien EM, Morgan BJ, Kozlowski MC. Perylenequinones: isolation, synthesis, and biological activity. Eur J Org Chem. 2012:21:3887-904.

5. O'Brien EM, Morgan BJ, Mulrooney CA, Carroll PJ, Kozlowski MC. Perylenequinone natural products: total synthesis of hypocrellin A. J Org Chem. 2010;75:57-68.

6. Cai YJ, Liang XH, Liao XR, Ding YR, Sun J, Li XH. High-yield hypocrellin A production in solid-state fermentation by Shiraia sp. SUPER-H168. Appl Biochem Biotechnol. 2010;160:2275-86.

7. Yang HL, Xiao C, Ma W, He G. The production of hypocrellin colorants by submerged cultivation of the medicinal fungus Shiraia bambusicola. Dyes Pigm. 2009;82:142-6.

8. Du W, Sun CL, Wang BG, Wang YM, Dong B, Liu JH, et al. Response mechanism of hypocrellin colorants biosynthesis by Shiraia bambusicola to elicitor PB90. AMB Expr. 2019;9:146.

9. Ma YJ, Zheng LP, Wang JW. Inducing perylenequinone production from a bambusicolous fungus Shiraia sp. S9 through co-culture with a fruiting body-associated bacterium Pseudomonas fulva SB1. Microb Cell Fact. 2019;18:121.

10. Lei $X Y$, Zhang MY, Ma YJ, Wang JW. Transcriptomic responses involved in enhanced production of hypocrellin A by addition of Triton X-100 in submerged cultures of Shiraia bambusicola. J Ind Microbiol Biotechnol. 2017:44:1415-29.

11. Li XP, Ma YJ, Wang JW. Adding bamboo charcoal powder to Shiraia bambusicola preculture improves hypocrellin A production. Sustain Chem Pharm. 2019;14:100191.

12. Du W, Liang JD, Han YF, Yu JP, Liang ZQ. Nitric oxide mediates hypocrellin accumulation induced by fungal elicitor in submerged cultures of Shiraia bambusicola. Biotechnol Lett. 2015;37:153-9.

13. Li XP, Wang Y, Ma YJ, Wang JW, Zheng LP. Nitric oxide and hydrogen peroxide signaling in extractive Shiraia fermentation by Triton $\mathrm{X}-100$ for hypocrellin A production. Int J Mol Sci. 2020;21:882. 
14. Tuteja N, Chandra M, Tuteja R, Misra MK. Nitric oxide as a unique bioactive signaling messenger in physiology and pathophysiology. J Biomed Biotechnol. 2004:4:227-37.

15. Zhang B, Zheng LP, Wang JW. Nitric oxide elicitation for secondary metabolite production in cultured plant cells. Appl Microbiol Biotechnol. 2012;93:455-66.

16. Farouk S, Al-Huqail AA. Sodium nitroprusside application regulates antioxidant capacity, improves phytopharmaceutical production and essential oil yield of marjoram herb under drought. Ind Crops Prod. 2020;158:113034

17. Wu CH, Tewari RK, Hahn EJ, Paek KY. Nitric oxide elicitation induces the accumulation of secondary metabolites and antioxidant defense in adventitious roots of Echinacea purpurea. J Plant Biol. 2007;50:636-43.

18. Zheng LP, Guo YT, Wang JW, Tan RX. Nitric oxide potentiates oligosaccharide- induced artemisinin production in Artemisia annua hairy roots. J Integr Plant Biol. 2008;50:49-55.

19. Wang JW, Zheng LP, Wu JY, Tan RX. Involvement of nitric oxide in oxidative burst, phenylalanine ammonia-lyase activation and taxol production induced by low-energy ultrasound in Taxus yunnanensis cell suspension cultures. Nitric Oxide. 2006;15:351-8.

20. Gong XY, Fu YP, Jiang DH, Li GQ, Yi XH, Peng YL. L-Arginine is essential for conidiation in the filamentous fungus Coniothyrium minitans. Fungal Genet Biol. 2007;44:1368-79.

21. Wang J, Higgins VJ. Nitric oxide has a regulatory effect in the germination of conidia of Colletotrichum coccodes. Fungal Genet Biol. 2005;42:284-92.

22. Baidya S, Cary JW, Grayburn WS, Calvo AM. Role of nitric oxide and flavohemoglobin homolog genes in Aspergillus nidulans sexual development and mycotoxin production. Appl Environ Microbiol. 2011;77:5524-8.

23. Zhao $Y X, X i$ Q, Xu Q, He MH, Ding JN, Dai YC, et al. Correlation of nitric oxide produced by an inducible nitric oxide synthase-like protein with enhanced expression of the phenylpropanoid pathway in Inonotus obliquus cocultured with Phellinus morii. Appl Microbiol Biotechnol. 2015:99:4361-72.

24. Gu L, Zhong X, Lian DH, Zheng YM, Wang HZ, Liu X. Triterpenoid biosynthesis and the transcriptional response elicited by nitric oxide in submerged fermenting Ganoderma lucidum. Process Biochem. 2017;60:19-26.

25. Sun CX, Ma YJ, Wang JW. Enhanced production of hypocrellin A by ultrasound stimulation in submerged cultures of Shiraia bambusicola. Ultrason Sonochem. 2017:38:214-24.

26. Wang JW, Zheng LP, Zhang B, Zou T. Stimulation of artemisinin synthesis by combined cerebroside and nitric oxide elicitation in Artemisia annua hairy roots. Appl Microbiol Biotechnol. 2009;85:285-92.

27. Yang HL, Wang Y, Zhang ZB, Yan RM, Zhu D. Whole-genome shotgun assembly and analysis of the genome of Shiraia sp. strain SIf14, a novel endophytic fungus producing huperzine A and hypocrellin A. Genome Announce. 2014;2:e00011-4.

28. Ren XY, Liu YX, Tan YM, Huang YH, Liu ZY, Jiang XL. Sequencing and functional annotation of the whole genome of Shiraia bambusicola. G3 Genes Genomes Genet. 2020;10:23-35.

29. Zhao N, Li D, Guo BJ, Tao X, Lin X, Yan SZ, et al. Genome sequencing and analysis of the hypocrellin-producing fungus Shiraia bambusicola S4201. Front Microbiol. 2020;11:643.

30. Ganjewala D, Bob S, Raghavendra AS. Sodium nitroprusside affects the level of anthocyanin and flavonol glycosides in pea (Pisum sativum L. CV Arkel) leaves. Acta Biol Szegediensis. 2008;52:301-5.

31. Khezerluo M, Hosseini B, Amiri J. Sodium nitroprusside stimulated production of tropane alkaloids and antioxidant enzymes activity in hairy root culture of Hyoscyamus reticulatus L. Acta Biol Hung. 2018;69:437-48.

32. Zhao YX, Lim JY, Xu JY, Yu JH, Zheng WF. Nitric oxide as a developmental and metabolic signal in filamentous fungi. Mol Microbiol. 2020;113:872-82.

33. Zhang Y, Shi HB, Liang S, Ning GA, Xu NC, Lu JP, et al. MoARG1, MoARG5,6 and MOARG7 involved in arginine biosynthesis are essential for growth, conidiogenesis, sexual reproduction, and pathogenicity in Magnaporthe oryzae. Microbiol Res. 2015;180:11-22.

34. Dong JF, Zhang M, Lu L, Sun LN, Xu MJ. Nitric oxide fumigation stimulates flavonoid and phenolic accumulation and enhances antioxidant activity of mushroom. Food Chem. 2012;135:1220-5.
35. Sun CX, Ma YJ, Wang JW. Improved hypocrellin A production in Shiraia bambusicola by light-dark shift. J Photochem Photobiol B Biol. 2018:182:100-7.

36. Ma YJ, Sun CX, Wang JW. Enhanced production of hypocrellin A in submerged cultures of Shiraia bambusicola by red light. Photochem Photobiol. 2019;95:812-22.

37. Du W, Liang ZQ, Zou X, Han YF, Liang JD, Yu JP, et al. Effects of microbial elicitor on production of hypocrellin by Shiraia bambusicola. Folia Microbiol. 2013;58:283-9.

38. Besson-Bard A, Pugin A, Wendehenne D. New insights into nitric oxide signaling in plants. Annu Rev Plant Biol. 2008;59:21-39.

39. Zheng LP, Zhang B, Zou T, Chen ZH, Wang JW. Nitric oxide interacts with reactive oxygen species to regulate oligosaccharide-induced artemisinin biosynthesis in Artemisia annua hairy roots. J Med Plants Res. 2010;4:758-66.

40. ligusa $H$, Yoshida $Y$, Hasunuma K. Oxygen and hydrogen peroxide enhance light-induced carotenoid synthesis in Neurospora crassa. FEBS Lett. 2005;579:4012-6.

41. Ponts N, Pinson-Gadais L, Verdal-Bonnin MN, Barreau C, Richard-Forget F. Accumulation of deoxynivalenol and its 15-acetylated form is significantly modulated by oxidative stress in liquid cultures of Fusarium graminearum. FEMS Microbiol Lett. 2006;258:102-7.

42. Reverberi M, Gazzetti K, Punelli F, Scarpari M, Zjalic S, Ricelli A, et al. Aoyap 1 regulates OTA synthesis by controlling cell redox balance in Aspergillus ochraceus. Appl Microbiol Biotechnol. 2012;95:1293-304.

43. Zhao N, Lin X, Qi SS, Luo ZM, Chen SL, Yan SZ. De novo transcriptome assembly in Shiraia bambusicola to investigate putative genes involved in the biosynthesis of hypocrellin A. Int J Mol Sci. 2016;17:311.

44. Hu JY, Sarrami F, Li H, Zhang GZ, Stubbs KA, Lacey E, et al. Heterologous biosynthesis of elsinochrome A sheds light on the formation of the photosensitive perylenequinone system. Chem Sci. 2019;10:1457-65.

45. Deng HX, Gao RJ, Liao XR, Cai YJ. Genome editing in Shiraia bambusicola using CRISPR-Cas9 system. J Biotechnol. 2017;259:228-34.

46. Li D, Zhao N, Guo BJ, Lin X, Chen SL, Yan SZ. Gentic overexpression increases production of hypocrellin A in Shiraia bambusicola S4201. J Microbiol. 2019;57:154-62.

47. Meraj TA, Fu JY, Raza MA, Zhu CY, Shen QQ, Xu DB, Wang Q. Transcriptional factors regulate plant stress responses through mediating secondary metabolism. Genes. 2020;11:346.

48. Beseli A, Amnuaykanjanasin A, Herrero S, Thomas E, Daub ME. Membrane transporters in self resistance of Cercospora nicotianae to the photoactivated toxin cercosporin. Curr Genet. 2015;61:601-20.

49. Ma YJ, Zheng LP, Wang JW. Bacteria associated with Shiraia fruiting bodies influence fungal production of hypocrellin A. Front Microbiol. 2019;10:2023.

50. Yao LY, Zhu YX, Jiao RH, Lu YH, Tan RX. Enhanced production of fumigaclavine $\mathrm{C}$ by ultrasound stimulation in a two-stage culture of Aspergillus fumigatus CY018. Bioresour Technol. 2014;159:112-7.

51. Turrion-Gomez JL, Benito EP. Flux of nitric oxide between the necrotrophic pathogen Botrytis cinerea and the host plant. Mol Plant Pathol. 2011;12:606-16.

52. Thevissen K, Terras FRG, Broekaert WF. Permeabilization of fungal membranes by plant defensins inhibits fungal growth. Appl Environ Microbiol. 1999;65:5451-8.

53. You BJ, Lee MH, Tien N, Lee MS, Hsieh HC, Tseng LH, et al. A novel approach to enhancing ganoderic acid production by Ganoderma lucidum using apoptosis induction. PLOS ONE. 2013;8:e53616.

54. Wu YX, Tiedemann AV. Impact of fungicides on active oxygen species and antioxidant enzymes in spring barley (Hordeum vulgare L.) exposed to ozone. Environ Pollut. 2002;116:37-47.

55. Pan WS, Zheng LP, Tian H, Li WY, Wang JW. Transcriptome responses involved in artemisinin production in Artemisia annua L under UV-B radiation. J Photochem Photobiol B Biol. 2014;140:292-300.

56. Aebi H. Catalase in vitro. Method Enzymol. 1984;105:121-6.

57. Marklund S, Marklund G. Involvement of the superoxide anion radical in the autoxidation of pyrogallol and a convenient assay for superoxide dismutase. Eur J Biochem. 1974;47:469-74.

58. Tongul B, Tarhan $L$. The effect of menadione-induced oxidative stress on the in vivo reactive oxygen species and antioxidant response system of Phanerochaete chrysosporium. Process Biochem. 2014;49:195-202. 
59. Lu CS, Ma YJ, Wang JW. Lanthanum elicitation on hypocrellin A production in mycelium cultures of Shiraia bambusicola is mediated by ROS generation. J Rare Earth. 2019;37:895-902.

60. Liu B, Bao JY, Zhang ZB, Yan RM, Wang Y, Yang HL, et al. Enhanced production of perylenequinones in the endophytic fungus Shiraia sp SIf14 by calcium/calmodulin signal transduction. Appl Microbiol Biotechnol. 2018:102:153-63.

61. Ma YJ, Lu CS, Wang JW. Effects of 5-azacytidine on growth and hypocrellin production of Shiraia bambusicola. Front Microbiol. 2018:9:2508.

62. Mortazavi A, Williams BA, McCue K, Schaeffer L, Wold B. Mapping and quantifying mammalian transcriptomes by RNA-Seq. Nat Methods. 2008;5:621-8.
63. Zhang J, Zhu LY, Chen HY, Li M, Zhu XJ, Gao Q, et al. A polyketide synthase encoded by the gene An15g07920 is involved in the biosynthesis of ochratoxin A in Aspergillus niger. J Agric Food Chem. 2016;64:9680-8.

\section{Publisher's Note}

Springer Nature remains neutral with regard to jurisdictional claims in published maps and institutional affiliations.
Ready to submit your research? Choose BMC and benefit from:

- fast, convenient online submission

- thorough peer review by experienced researchers in your field

- rapid publication on acceptance

- support for research data, including large and complex data types

- gold Open Access which fosters wider collaboration and increased citations

- maximum visibility for your research: over $100 \mathrm{M}$ website views per year

At BMC, research is always in progress.

Learn more biomedcentral.com/submissions 